\title{
Mass, momentum, and energy flux conservation for nonlinear wave-wave interaction
}

\author{
Zhen Liu, ${ }^{1, a), b)}$ Zhiliang Lin, ${ }^{1,2, b), c)}$ and Longbin Tao ${ }^{3, d)}$ \\ ${ }^{1}$ State Key Laboratory of Ocean Engineering, Shanghai Jiao Tong University, \\ 800 Dongchuan Road, Shanghai 200240, China \\ ${ }^{2}$ Collaborative Innovation Center for Advanced Ship and Deep-Sea Exploration (CISSE), \\ Shanghai Jiao Tong University, 800 Dongchuan Road, Shanghai 200240, China \\ ${ }^{3}$ School of Marine Science and Technology, Newcastle University, Armstrong Building, \\ Newcastle upon Tyne NE1 7RU, United Kingdom
}

(Received 15 July 2016; accepted 15 November 2016; published online 8 December 2016)

\begin{abstract}
A fully nonlinear solution for bi-chromatic progressive waves in water of finite depth in the framework of the homotopy analysis method (HAM) is derived. The bi-chromatic wave field is assumed to be obtained by the nonlinear interaction of two monochromatic wave trains that propagate independently in the same direction before encountering. The equations for the mass, momentum, and energy fluxes based on the accurate high-order homotopy series solutions are obtained using a discrete integration and a Fourier series-based fitting. The conservation equations for the mean rates of the mass, momentum, and energy fluxes before and after the interaction of the two nonlinear monochromatic wave trains are proposed to establish the relationship between the steady-state bi-chromatic wave field and the two nonlinear monochromatic wave trains. The parametric analysis on $\varepsilon_{1}$ and $\varepsilon_{2}$, representing the nonlinearity of the bi-chromatic wave field, is performed to obtain a sufficiently small standard deviation $S_{d}$, which is applied to describe the deviation from the conservation state $\left(S_{d}=0\right)$ in terms of the mean rates of the mass, momentum, and energy fluxes before and after the interaction. It is demonstrated that very small standard deviation from the conservation state can be achieved. After the interaction, the amplitude of the primary wave with a lower circular frequency is found to decrease; while the one with a higher circular frequency is found to increase. Moreover, the highest horizontal velocity of the water particles underneath the largest wave crest, which is obtained by the nonlinear interaction between the two monochromatic waves, is found to be significantly higher than the linear superposition value of the corresponding velocity of the two monochromatic waves. The present study is helpful to enrich and deepen the understanding with insight to steady-state wave-wave interactions. Published by AIP Publishing. [http://dx.doi.org/10.1063/1.4971252]
\end{abstract}

\section{INTRODUCTION}

Ocean surface waves are irregular and intuitively viewed as a superposition of many monochromatic wave components of different frequencies and amplitudes. Nonlinear interactions among these wave components are very important to resultant wave properties. During the past several decades, a considerable number of studies have been carried out to analyze nonlinear wave interaction theories. Phillips ${ }^{1}$ and Longuet-Higgins ${ }^{2}$ initially revealed the resonant phenomenon obtained from nonlinear interactions between two or three wave trains. It was pointed out that, under specific conditions, conspicuous energy transfer occurs from primary waves to a tertiary wave, produced via

\footnotetext{
a)Electronic mail: liuzhen0829@sjtu.edu.cn

b) Z. Liu and Z. Lin contributed equally to this work.

c) Electronic mail: linzhiliang@ @jtu.edu.cn

d) Author to whom correspondence should be addressed. Electronic mail: longbin.tao@newcastle.ac.uk
} 
the third-order interaction. Pierson ${ }^{3}$ derived an oscillatory third-order perturbation solution for two and three collinear interacting Stokes waves in deep water. However, Madsen and Fuhrman ${ }^{8}$ pointed out that the dispersion relation obtained by Pierson ${ }^{3}$ was not based on consistent perturbation principles and thus is incorrect, and they further presented a new third-order solution for bi-chromatic bi-directional water waves in finite depth, extending the second-order in finite depth and third-order in infinite depth theories of steady bi-chromatic waves. Their solution includes explicit expressions for the surface elevation, the amplitude dispersion, and the velocity potential. Dalzell ${ }^{4}$ employed symbolic computation to extend the second-order wave-wave interaction theory from deep water to finite water depth. Ohyama et al..$^{5}$ obtained a fourth-order solution for nonlinear interactions among multiple directional wave trains by using a Stokes-type expansion method. It was indicated that the third- and fourth-order components may produce isolated large crests in random wave fields. Chen and Zhang ${ }^{6}$ studied the interaction between a unidirectional deep-water short-wave train and an intermediate water-depth long wave using a conventional perturbation method and a phase modulation method, respectively. It was revealed that the modulation of the short-wave intrinsic frequency and potential amplitude along the long-wave surface becomes significant as water depth decreases, together with the increasing modulation of the short-wave phase, amplitude, and wave number. Zhang and $\mathrm{Chen}^{7}$ further derived a general third-order analytical solution for the strong interactions among three collinear free-wave components using a perturbation method, and this solution is regarded as the kernel of third-order collinear irregular wave theory.

Most of the aforementioned studies are based on the perturbation technique due to its solid mathematical foundation on the basis of the asymptotic expansion with respect to some small parameters. As the nonlinearity increases, in order to obtain accurate results, higher-order solutions are required. However, the derivation of the higher-order perturbation solution for nonlinear wave-wave interaction problems can be lengthy and very complex.

Jang and Kwon ${ }^{9}$ proposed a fixed point approach to calculate nonlinear monochromatic wave profiles and later Jang et al..$^{10}$ apply to evaluate the nonlinear wave profiles of wave-wave interactions in a finite water depth. It is worth noting that the results by Jang et al. ${ }^{10}$ do not satisfy the exact kinematic and dynamic free surface boundary conditions and thus fail to capture strongly nonlinear features. To evaluate the strongly nonlinear characteristics of wave-wave interaction, Lin et al. ${ }^{11}$ investigated fully nonlinear bi-chromatic unidirectional waves propagating in deep water using the so-called homotopy analysis method (HAM). The particular advantage of HAM is that it is independent of small parameters and suitable to solve strongly nonlinear problems. Other advantages associated with HAM include a greater flexibility in the selection of a proper set of base functions for the solution and a simple way in the control of the convergence rate and region of solution series. $^{12}$

HAM was first applied to monochromatic, progressive waves in deep water by Liao and Cheung. ${ }^{13}$ Later, Tao et al. ${ }^{14}$ successfully extended Liao and Cheung ${ }^{13}$ to waters of finite depth. More recently, Liao ${ }^{15}$ proposed a multiple-variable technique to investigate steady-state resonant progressive waves in deep water in the framework of HAM. It is demonstrated that there exist multiple resonant waves, and that the amplitudes of resonant wave may be much smaller than those of primary waves thus the resonant waves sometimes contain fairly small part of wave energy. Xu et al. ${ }^{16}$ further confirmed the existence of steady-state resonant progressive waves in finite water depth by means of HAM and obtained qualitatively identical conclusion using the Zakharov equation. Liu and $\mathrm{Liao}^{17}$ extended the existing results of Liao ${ }^{15}$ and Xu et al. ${ }^{16}$ on steady-state resonance from a single special quartet to more general and coupled resonant quartets, as well as a resonant sextet. The aforementioned studies on steady-state wave resonance are based on the assumption that all of the wave amplitudes, wave numbers, and wave frequencies are independent of time in the wave system. To date, however, whether or not HAM can be applied to address the unsteady-state wave resonance involving complicated issues, which can account for the wave instability phenomena ${ }^{18}$ (e.g., modulational instability, also known as Benjamin-Feir instability), deserves further investigation.

Most of the aforementioned studies were focused on the resultant wave field produced by the interaction between two or multiple progressive wave components. The relationship between the resultant wave field and the monochromatic progressive waves before the interaction has not yet been discussed. Neglecting viscous dissipation, Baddour and Song ${ }^{19,20}$ introduced conservation 
equations for the mean rates of the mass, momentum, and energy fluxes before and after the interaction between collinear current-free monochromatic waves and a wave-free current based on a perturbation method. Zaman and Baddour ${ }^{21}$ further extended the work by Baddour and Song ${ }^{19,20}$ to a three-dimensional flow frame. However, the interaction of two nonlinear monochromatic progressive wave components which propagate independently before encountering, in terms of the conservation equations for the mass, momentum, and energy fluxes, has not been considered. In this paper, by including constant water depth in the solution procedure, the present study extends the work of Lin et al. ${ }^{11}$ from infinite water depth to finite water depth. Furthermore, based on the assumption that the steady-state bi-chromatic wave system can be obtained by the interaction of two nonlinear monochromatic progressive wave trains which propagate independently in the same direction before encountering, the present study aims to establish the relationship between the steady bi-chromatic wave field and the two nonlinear monochromatic progressive wave trains, in terms of the conservation equations for the mean rates of the mass, momentum, and energy fluxes before and after the interaction, respectively.

The present paper is organized as follows. The mathematical description of the bi-chromatic wave field is given in Sec. II following the Introduction. The definitions of the equations for the mass, momentum, and energy fluxes are introduced in Sec. III. The conservation equations for the wave-wave interaction are presented in Sec. IV. The detailed results on the parametric analysis for the standard deviation from the conservation state $\left(S_{d}=0\right)$, as well as the characteristics of the bi-chromatic wave field with sufficiently small values for $S_{d}$, which is defined to describe the deviation from the conservation state before and after interaction, are discussed in Sec. V. Finally, conclusions are given, with the detailed solution procedure based on HAM in the Appendix.

\section{MATHEMATICAL DESCRIPTION OF THE BI-CHROMATIC WAVE FIELD}

\section{A. Basic equations}

Fig. 1 shows the definition sketch for a steady-state bi-chromatic wave field which is assumed to be produced by the interaction of two nonlinear, monochromatic, progressive wave components that propagate independently in the same direction before interacting. A Cartesian coordinate system $(x, z)$ is adopted where the $x$-axis is positive in the direction of wave propagation, and the $z$-axis is positive vertically upwards from the still water level as shown in Fig. 1. It is assumed that the nonlinear monochromatic wave train with a higher phase velocity will catch up to and interact thoroughly with the one with a lower phase velocity, yielding the steady-state bi-chromatic wave

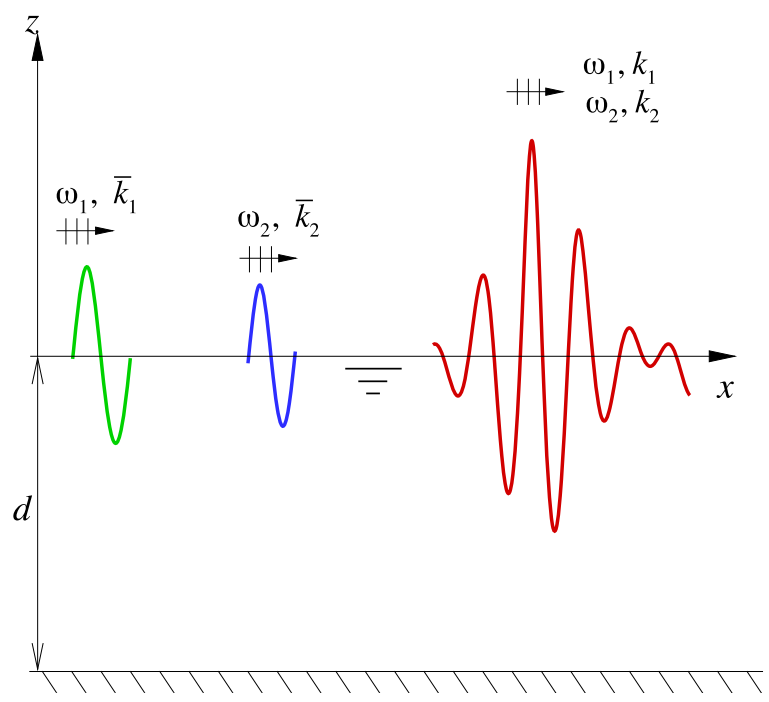

FIG. 1. Definition sketch for a steady-state bi-chromatic wave field. 
field. For the bi-chromatic wave field, the fluid considered is inviscid and incompressible, and the flow is assumed to be irrotational. The quantities $\varphi(x, z, t)$ and $\zeta(x, t)$ are defined as the velocity potential and the wave elevation, respectively. The fluid motion described by the velocity potential $\varphi$ is governed by the Laplace equation,

$$
\nabla^{2} \varphi(x, z, t)=0, \quad-\infty<x<+\infty,-d<z<\zeta(x, t),
$$

and subject to two nonlinear free surface conditions,

$$
\begin{gathered}
\frac{\partial \zeta}{\partial t}+\frac{\partial \varphi}{\partial x} \frac{\partial \zeta}{\partial x}-\frac{\partial \varphi}{\partial z}=0, \quad z=\zeta(x, t), \\
g \zeta+\frac{1}{2}(\nabla \varphi) \cdot(\nabla \varphi)+\frac{\partial \varphi}{\partial t}=0, \quad z=\zeta(x, t),
\end{gathered}
$$

and the following condition at the bottom:

$$
\frac{\partial \varphi}{\partial z}=0, \quad z=-d,
$$

where $\nabla=(\partial / \partial x, \partial / \partial z), t$ denotes time, $g$ is the gravitational acceleration, and $d$ is the water depth. Since gravity capillary waves caused by surface tension are quite small compared to their wavelengths, the effect of surface tension is neglected.

Combining Eqs. (2) and (3), the free surface boundary condition becomes

$$
\frac{\partial^{2} \varphi}{\partial t^{2}}+g \frac{\partial \varphi}{\partial z}+\frac{\partial[(\nabla \varphi) \cdot(\nabla \varphi)]}{\partial t}+\frac{1}{2}(\nabla \varphi) \cdot \nabla[(\nabla \varphi) \cdot(\nabla \varphi)]=0, \quad z=\zeta(x, t) .
$$

\section{B. Multiple-variable transformation}

The frequencies and wave numbers of the primary waves of the bi-chromatic wave field are defined by $\omega_{i}$ and $k_{i}(i=1,2)$, respectively. It is convenient to define the phase functions,

$$
\begin{aligned}
& \theta_{1}=k_{1} x-\omega_{1} t+\Phi_{1}, \\
& \theta_{2}=k_{2} x-\omega_{2} t+\Phi_{2},
\end{aligned}
$$

where $\Phi_{i}(i=1,2)$ denotes an arbitrary, constant phase for zero time at the origin of the $x-z$ coordinate system. Whilst $k_{1} \omega_{2} \neq k_{2} \omega_{1}$, the above two variables can be applied to replace the variables $x$ and $t$, and then the time $t$ will not appear explicitly for a steady-state wave system. Thus, the potential function and wave elevation for the steady-state bi-chromatic wave field can be expressed as $\varphi(x, z, t)=\phi\left(\theta_{1}, \theta_{2}, z\right)$ and $\zeta(x, t)=\eta\left(\theta_{1}, \theta_{2}\right)$, respectively. With these definitions, the governing equation becomes

$$
\hat{\nabla}^{2} \phi=k_{1}^{2} \frac{\partial^{2} \phi}{\partial \theta_{1}^{2}}+2 k_{1} k_{2} \frac{\partial^{2} \phi}{\partial \theta_{1} \partial \theta_{2}}+k_{2}^{2} \frac{\partial^{2} \phi}{\partial \theta_{2}^{2}}+\frac{\partial^{2} \phi}{\partial z^{2}}=0, \quad-d<z<\eta\left(\theta_{1}, \theta_{2}\right),
$$

which is subject to the bottom boundary condition,

$$
\frac{\partial \phi}{\partial z}=0, \quad z=-d,
$$

and the nonlinear free surface conditions,

$$
\begin{gathered}
\eta=\frac{1}{g}\left(\omega_{1} \frac{\partial \phi}{\partial \theta_{1}}+\omega_{2} \frac{\partial \phi}{\partial \theta_{2}}-f\right), \quad z=\eta\left(\theta_{1}, \theta_{2}\right), \\
\omega_{1}^{2} \frac{\partial^{2} \phi}{\partial \theta_{1}^{2}}+2 \omega_{1} \omega_{2} \frac{\partial^{2} \phi}{\partial \theta_{1} \partial \theta_{2}}+\omega_{2}^{2} \frac{\partial^{2} \phi}{\partial \theta_{2}^{2}}+g \frac{\partial \phi}{\partial z} \\
-2\left(\omega_{1} \frac{\partial f}{\partial \theta_{1}}+\omega_{2} \frac{\partial f}{\partial \theta_{2}}\right)+\hat{\nabla} \phi \cdot \hat{\nabla} f=0, \quad z=\eta\left(\theta_{1}, \theta_{2}\right),
\end{gathered}
$$


where

$$
\begin{gathered}
f=\frac{1}{2}\left[k_{1}^{2}\left(\frac{\partial \phi}{\partial \theta_{1}}\right)^{2}+2 k_{1} k_{2} \frac{\partial \phi}{\partial \theta_{1}} \frac{\partial \phi}{\partial \theta_{2}}+k_{2}^{2}\left(\frac{\partial \phi}{\partial \theta_{2}}\right)^{2}+\left(\frac{\partial \phi}{\partial z}\right)^{2}\right] \\
\text { and } \hat{\nabla}=\left(k_{1} \partial / \partial \theta_{1}+k_{2} \partial / \partial \theta_{2}, \partial / \partial z\right) .
\end{gathered}
$$

Due to the nonlinear interaction, the wave elevation should be in the form

$$
\eta\left(\theta_{1}, \theta_{2}\right)=\sum_{m=0}^{+\infty} \sum_{n=-\infty}^{+\infty} a_{m, n} \cos \left(m \theta_{1}+n \theta_{2}\right),
$$

and the corresponding potential function should be in the form

$$
\phi\left(\theta_{1}, \theta_{2}, z\right)=\sum_{m=0}^{+\infty} \sum_{n=-\infty}^{+\infty} b_{m, n} \Psi_{m, n}\left(\theta_{1}, \theta_{2}, z\right)
$$

where

$$
\Psi_{m, n}\left(\theta_{1}, \theta_{2}, z\right)=\sin \left(m \theta_{1}+n \theta_{2}\right) \frac{\cosh \left[\left|m k_{1}+n k_{2}\right|(z+d)\right]}{\cosh \left[\left|m k_{1}+n k_{2}\right| d\right]},
$$

and $a_{m, n}, b_{m, n}$ are constants to be determined. It should be noted that (15) automatically satisfies the governing Eq. (8) and the bottom boundary condition (9).

\section{Solution procedures}

As a first step to consider the nonlinear effects on the steady-state bi-chromatic waves in finite water depth, it is assumed that the nonlinear dispersion relation in the wave system can be described as $\omega_{i}=\varepsilon_{i} \sqrt{g k_{i} \tanh \left(k_{i} d\right)}(i=1,2)$, where $\varepsilon_{i}$ is a parameter slightly larger than 1 , representing the nonlinearity of the wave system. As long as $\varepsilon_{i}, \omega_{i}$, and $d$ are given, $k_{i}$ can be easily obtained by the nonlinear dispersion relation. Once $\omega_{i}, k_{i}$, and $d$ are known, it is not difficult to obtain $a_{m, n}$ and $b_{m, n}$ by HAM.

Lin et al. ${ }^{11}$ successfully applied HAM to obtain a high-order series solution for deep-water bi-chromatic progressive waves. The effectiveness of HAM for wave-wave interaction was validated by Lin et al. ${ }^{11}$ by comparing the HAM solutions for the wave profile and water particle velocity with those obtained based on the perturbation technique. For the sake of simplicity, a brief description of the solution procedure in the framework of HAM is provided in the Appendix. It is worth noting that the HAM solution procedure for each nonlinear monochromatic wave train is similar to that for the bi-chromatic waves, and the detailed HAM solution procedure for monochromatic, progressive waves can also be found in the works of Liao and Cheung ${ }^{13}$ and Tao et al. ${ }^{14}$

\section{Validation of the analytical model}

The present series solution for bi-chromatic progressive waves in finite water depth is validated

\begin{tabular}{|c|c|c|c|c|c|c|c|}
\hline \multirow[b]{2}{*}{ Case } & \multicolumn{2}{|c|}{$\begin{array}{l}\text { Experimental (Ma } \\
\text { et al. }{ }^{22} \text { ) and HAM }\end{array}$} & \multicolumn{2}{|c|}{$\begin{array}{l}\text { Experimental (Ma } \\
\left.\quad \text { et al. }^{22}\right)\end{array}$} & \multicolumn{2}{|c|}{ HAM } & \multirow[b]{2}{*}{$\mathrm{E}_{m}^{T}(m=10)$} \\
\hline & $f_{1}(\mathrm{~Hz})$ & $f_{2}(\mathrm{~Hz})$ & $a_{1}(\mathrm{~m})$ & $a_{2}(\mathrm{~m})$ & $a_{1,0}(\mathrm{~m})$ & $a_{0,1}(\mathrm{~m})$ & \\
\hline 1 & 0.9 & 1.1 & 0.0185 & 0.0205 & 0.018541 & 0.020487 & $1.15 \times 10^{-8}$ \\
\hline 2 & 0.875 & 1.125 & 0.0185 & 0.0205 & 0.018526 & 0.021357 & $1.31 \times 10^{-7}$ \\
\hline 3 & 0.85 & 1.15 & 0.0185 & 0.0205 & 0.018452 & 0.021344 & $3.64 \times 10^{-6}$ \\
\hline 4 & 0.825 & 1.175 & 0.0185 & 0.0205 & 0.018555 & 0.026647 & $1.30 \times 10^{-4}$ \\
\hline
\end{tabular}
by comparison to experimental data of Ma et al..$^{22}$ Table I shows the parameters of the bi-chromatic wave cases in the experiments of Ma et al. ${ }^{22}$ and corresponding results obtained by HAM. As shown in Table I, for the identical frequencies $\left(f_{1}\right.$ and $\left.f_{2}\right)$ of primary waves of each case (cases $\left.1-4\right)$ in the

TABLE I. Parameters of bi-chromatic waves $(d=0.5 \mathrm{~m})$. 
experiments and HAM solutions, the amplitudes of primary waves $\left(a_{1,0}\right.$ and $\left.a_{0,1}\right)$ obtained by HAM have slight differences with those $\left(a_{1}\right.$ and $\left.a_{2}\right)$ in the experiments, respectively. This is attributed to the nonlinear characteristics of the HAM solution. Moreover, the total averaged residual square error $\left(\mathrm{E}_{m}^{T}\right)$ of cases 1-4 returns a fairly small value, which reaches at least the order of magnitude of $10^{-4}$. This indicates that the HAM solution for cases 1-4 possesses higher accuracy. Fig. 2 shows

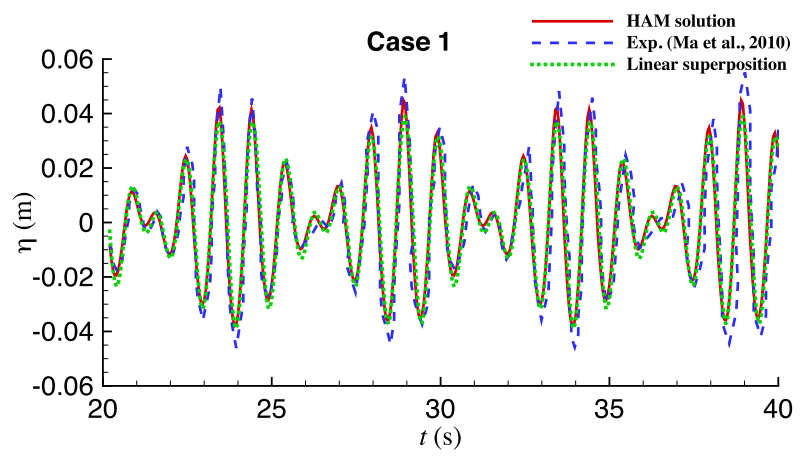

(a)

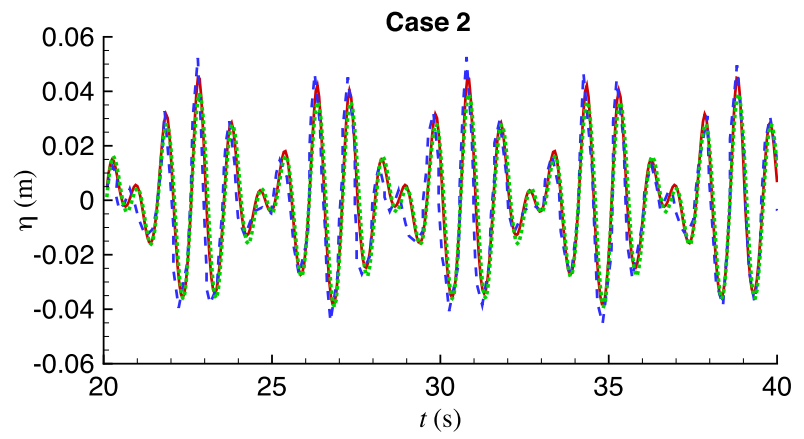

(b)
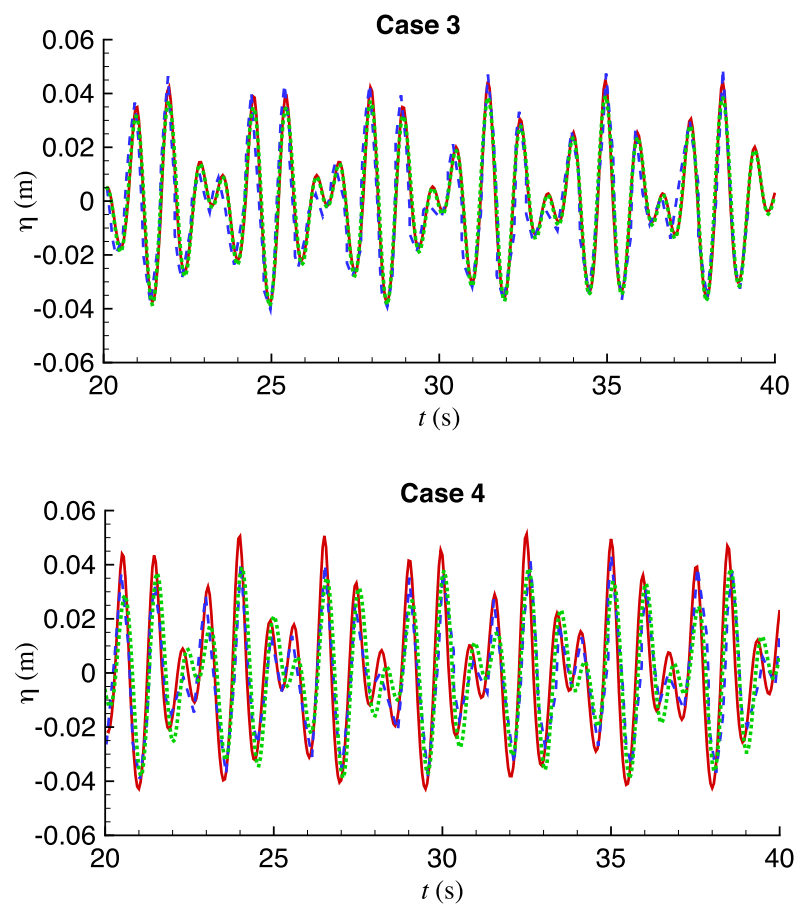

(d)

FIG. 2. Comparisons of time series of wave elevation between the HAM solutions, the experimental data, and corresponding linear superposition results. 
TABLE II. Parameters for the study of nonlinear amplitude dispersion $\left(k_{1} d=2, k_{2} d=2.86\right)$.

\begin{tabular}{|c|c|c|c|c|c|c|c|}
\hline \multirow[b]{2}{*}{ Case } & \multirow[b]{2}{*}{$a_{1,0}(\mathrm{~m})$} & \multirow[b]{2}{*}{$a_{0,1}(\mathrm{~m})$} & \multicolumn{2}{|c|}{$\begin{array}{c}\text { 3rd-order perturbation } \\
\text { (Madsen and Fuhrman }^{8} \text { ) }\end{array}$} & \multicolumn{2}{|c|}{ HAM } & \multirow[b]{2}{*}{$\mathrm{E}_{m}^{T}(m=10)$} \\
\hline & & & $\omega_{1} / \bar{\omega}_{1}$ & $\omega_{2} / \bar{\omega}_{2}$ & $\omega_{1} / \bar{\omega}_{1}$ & $\omega_{2} / \bar{\omega}_{2}$ & \\
\hline A1 & 0.052989 & 0.14654 & 1.001 & 1.00101 & 1.001 & 1.001 & $1.42 \times 10^{-7}$ \\
\hline $\mathrm{A} 2$ & 0.07738 & 0.205276 & 1.00198 & 1.00201 & 1.002 & 1.002 & $2.08 \times 10^{-7}$ \\
\hline A3 & 0.115589 & 0.284759 & 1.00384 & 1.00394 & 1.004 & 1.004 & $2.14 \times 10^{-7}$ \\
\hline A4 & 0.14787 & 0.34188 & 1.00559 & 1.00579 & 1.006 & 1.006 & $1.86 \times 10^{-7}$ \\
\hline A5 & 0.176573 & 0.386578 & 1.00722 & 1.00755 & 1.008 & 1.008 & $8.32 \times 10^{-7}$ \\
\hline A6 & 0.202365 & 0.42256 & 1.00871 & 1.00918 & 1.01 & 1.01 & $8.36 \times 10^{-7}$ \\
\hline A7 & 0.224841 & 0.451334 & 1.01002 & 1.01064 & 1.012 & 1.012 & $6.09 \times 10^{-7}$ \\
\hline A8 & 0.244576 & 0.473958 & 1.01114 & 1.01191 & 1.014 & 1.014 & $8.22 \times 10^{-7}$ \\
\hline A9 & 0.261417 & 0.490857 & 1.01204 & 1.01296 & 1.016 & 1.016 & $9.12 \times 10^{-6}$ \\
\hline A10 & 0.275165 & 0.502195 & 1.01269 & 1.01375 & 1.018 & 1.018 & $7.25 \times 10^{-5}$ \\
\hline
\end{tabular}

the time series of wave elevation for cases 1-4 by the HAM solution, together with the experimental data by Ma et al..$^{22}$ and corresponding linear superposition results. It can be clearly seen in Fig. 2 that, in comparison to the linear superposition results, the HAM solution demonstrates a much better agreement with the experimental data. This further verifies the effectiveness of the present series solution.

Lin et al. ${ }^{11}$ compared the nonlinear amplitude dispersion for bi-chromatic unidirectional waves in deep water obtained by HAM to the 3rd-order perturbation results by Madsen and Fuhrman. ${ }^{8}$ It is demonstrated that, for the bi-chromatic waves with identical amplitude $\left(a_{1,0}=a_{0,1}\right)$ of the two primary wave components and different wave numbers $\left(k_{1}, k_{2}\right)=(0.3,0.4)$, the HAM solutions agree well with the perturbation results when $k_{1} a_{1,0}<0.045$ (or $k_{2} a_{0,1}<0.06$ ) and exhibit a relatively evident misalignment with the perturbation results when $k_{1} a_{1,0}>0.045$. In this paper, to further validate the effectiveness of the present series solution, the nonlinear amplitude dispersions for interacting bi-chromatic unidirectional waves in finite water depth by HAM are compared to those by Madsen and Fuhrman. ${ }^{8}$ Table II shows the parameters for the study of nonlinear amplitude dispersion.

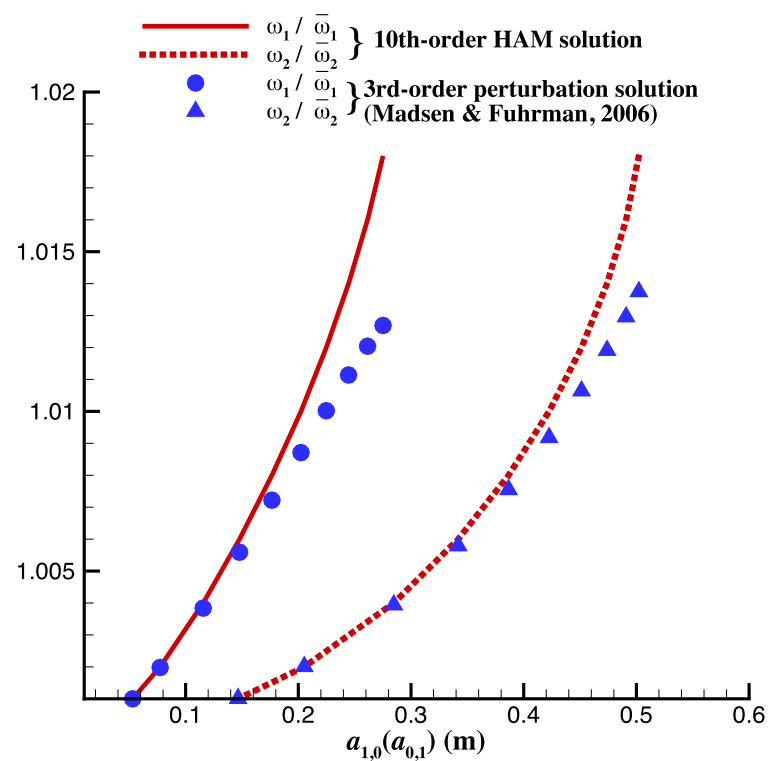

FIG. 3. Comparison of nonlinear amplitude dispersion between HAM solutions and perturbation results by Madsen and Fuhrman. ${ }^{8}$ 


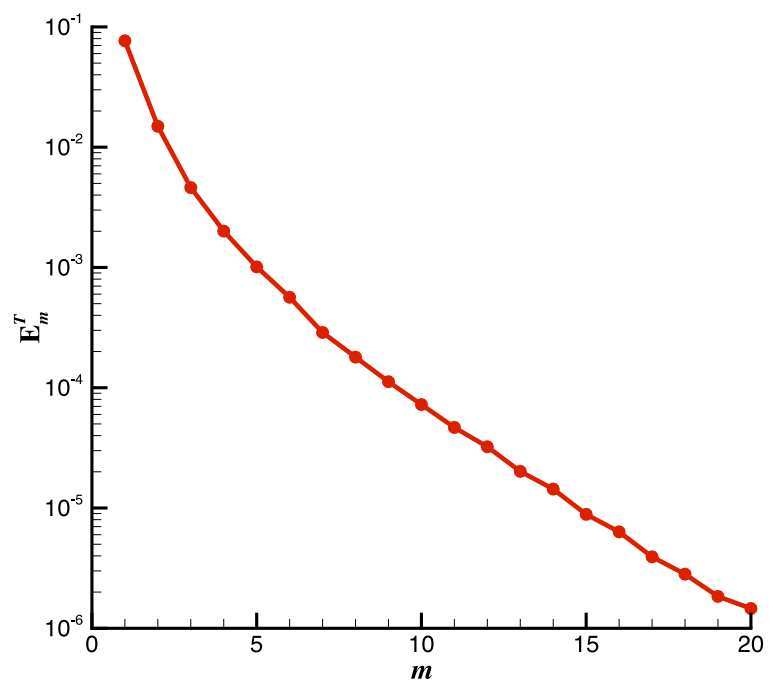

FIG. 4. The total averaged residual square error $\mathrm{E}_{m}^{T}$ versus $m$ with $c_{0}=-1$ in case A10.

The wave numbers of each case of Table II in the HAM solution and the perturbation solution are $\left(k_{1}, k_{2}\right)=(0.2,0.285714)$. The amplitudes of primary waves $\left(a_{1,0}\right.$ and $\left.a_{0,1}\right)$ of each case in the HAM solution and the perturbation solution are increasing gradually from cases A1 to A10, respectively, indicating the increasing nonlinearity of the bi-chromatic wave system. It is noted that the relative

(a)

$$
\text { Case BW, } \varepsilon_{1}=\varepsilon_{2}=1.008
$$

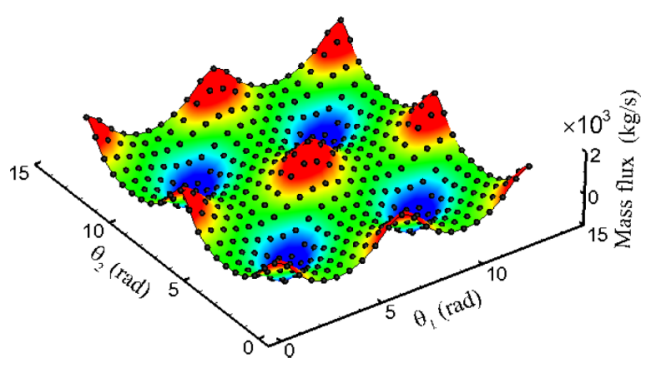

(b)

Case BW, $\varepsilon_{1}=\varepsilon_{2}=1.008$

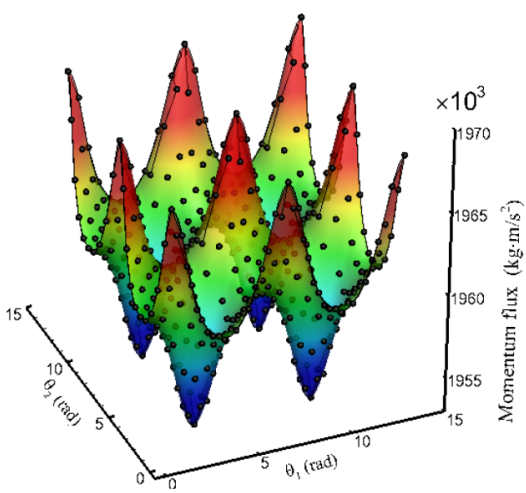

(c)

Case BW, $\varepsilon_{1}=\varepsilon_{2}=1.008$

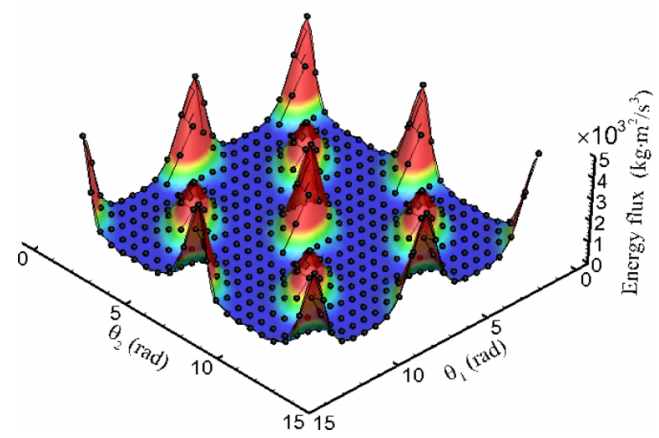

FIG. 5. The comparison of the discrete integral value points and fitted function curved surfaces for: (a) mass flux; (b) momentum flux; (c) energy flux (Filled circle: the discrete integrations; curved surface: the fitted functions) of the case BW with $\varepsilon_{1}=\varepsilon_{2}=1.008$. 
water depths of the two primary wave components are $k_{1} d=2$ and $k_{2} d=2.86$, respectively, corresponding to intermediate water depth conditions. As shown in Table II, the values for the relative nonlinear frequencies $\omega_{1} / \bar{\omega}_{1}$ and $\omega_{2} / \bar{\omega}_{2}$ obtained by HAM agree well with the 3rd-order perturbation results from cases A1 to A5 and have relatively large discrepancies from cases A6 to A10.

To clearly see the tendency of the nonlinear amplitude dispersion, $\omega_{1} / \bar{\omega}_{1}$ and $\omega_{2} / \bar{\omega}_{2}$ are plotted against $a_{1,0}$ and $a_{0,1}$ in Fig. 3, respectively. It can be evidently seen that the present HAM solution appears to be different with the perturbation results starting from case A6 $\left(a_{1,0}=0.202365, a_{0,1}=\right.$ 0.42256 ). The total averaged residual square error for cases A6-A10 reaches at least the order of magnitude of $10^{-5}$, indicating that the present HAM solutions are highly accurate. To further demonstrate the convergence of the HAM solution, Fig. 4 shows the total averaged residual square error $\mathrm{E}_{m}^{T}$ versus $m$ with $c_{0}=-1$ in case A10. It can be seen that even for this rather strongly nonlinear case (A10), $\mathrm{E}_{m}^{T}$ decreases gradually to the order of magnitude of $10^{-6}$ as $m$ increases from 1 to 20, a clear indication of convergence of the HAM solution for this case.

\section{DEFINITION OF MASS, MOMENTUM, AND ENERGY FLUX EQUATIONS}

Similar to the flux equations in Whitham, ${ }^{23}$ the mean rates of the mass, momentum, and energy fluxes across a vertical section fixed in the bi-chromatic wave field, denoted by $Q^{B W}, M^{B W}$, and $E^{B W}$ respectively, can be written as

$$
\begin{gathered}
Q^{B W}=\frac{1}{4 \pi^{2}} \int_{0}^{2 \pi} \int_{0}^{2 \pi} \int_{-d}^{\eta} \rho \phi_{x} \mathrm{~d} z \mathrm{~d} \theta_{1} \mathrm{~d} \theta_{2}, \\
M^{B W}=\frac{1}{4 \pi^{2}} \int_{0}^{2 \pi} \int_{0}^{2 \pi} \int_{-d}^{\eta}\left(P+\rho \phi_{x}^{2}\right) \mathrm{d} z \mathrm{~d} \theta_{1} \mathrm{~d} \theta_{2}, \\
E^{B W}=\frac{1}{4 \pi^{2}} \int_{0}^{2 \pi} \int_{0}^{2 \pi} \int_{-d}^{\eta}\left[P+\frac{\rho}{2}\left(\phi_{x}^{2}+\phi_{z}^{2}\right)+\rho g z\right] \phi_{x} \mathrm{~d} z \mathrm{~d} \theta_{1} \mathrm{~d} \theta_{2},
\end{gathered}
$$

where $\rho$ denotes density of water, and $P$ is the total pressure which can be determined by the Bernoulli equation for the wave field as

$$
\frac{P}{\rho}=-\frac{\partial \phi}{\partial t}-\frac{1}{2}(\nabla \phi)^{2}-g z
$$

Different from the method for computing the integral quantities of the mass, momentum, and energy fluxes by means of low-order perturbation approximations by Whitham, ${ }^{23}$ Baddour and Song, ${ }^{19,20}$ and Zaman and Baddour, ${ }^{21}$ the accurate high-order homotopy series solutions for the pressure, water particle velocity, and free surface elevation are employed to calculate the corresponding integrations for the present bi-chromatic wave cases, i.e., $Q^{B W}, M^{B W}$, and $E^{B W}$. Due to the complex

\begin{tabular}{|c|c|c|c|c|c|c|}
\hline \multirow[b]{2}{*}{$f_{h, l}$} & & \multicolumn{5}{|c|}{$h$} \\
\hline & & 0 & 1 & 2 & 3 & 4 \\
\hline \multirow{9}{*}{$l$} & -4 & 0.000397 & 0.00381422 & 0.013113 & 0.0230707 & -0.00125484 \\
\hline & -3 & 0.002415 & 0.0260936 & 0.0674599 & -0.00109471 & -0.000143365 \\
\hline & -2 & 0.021295 & 0.231307 & -0.00160349 & 0.00763481 & 0.00100312 \\
\hline & -1 & 0.349504 & -0.00320619 & 0.0851259 & 0.00393892 & 0.000593725 \\
\hline & 0 & 0.0438276 & 0.56839 & 0.0182248 & 0.00127507 & 0.000381219 \\
\hline & 1 & 0.349504 & 0.0400968 & 0.00288494 & 0.000442871 & 0.000326527 \\
\hline & 2 & 0.021295 & 0.0045469 & 0.000559225 & 0.000288311 & 0.000386793 \\
\hline & 3 & 0.002415 & 0.000731839 & 0.000245947 & 0.000423822 & 0.000788489 \\
\hline & 4 & 0.000397 & 0.000257311 & 0.000498968 & 0.00137073 & 0.00249853 \\
\hline
\end{tabular}
integrands and integral upper limit incorporating the variables $x$ and $t$, it is difficult to obtain these integral quantities by direct integrating. Thus, the phase function $\theta_{i}=k_{i} x-\omega_{i} t(i=1,2)$ is applied

TABLE III. The coefficients for the fitted function $F_{Q}\left(\theta_{1}, \theta_{2}\right)$ for the mass flux of the case BW with $\varepsilon_{1}=\varepsilon_{2}=1.008$. 
TABLE IV. The coefficients for the fitted function $F_{M}\left(\theta_{1}, \theta_{2}\right)$ for the momentum flux of the case BW with $\varepsilon_{1}=\varepsilon_{2}=1.008$.

\begin{tabular}{|c|c|c|c|c|c|c|}
\hline \multirow[b]{2}{*}{$f_{h, l}$} & & \multicolumn{3}{|c|}{$h$} & \multirow[b]{2}{*}{3} & \multirow[b]{2}{*}{4} \\
\hline & & 0 & 1 & 2 & & \\
\hline \multirow{9}{*}{$l$} & -4 & 0.001678 & 0.0161032 & 0.0535568 & 0.0849963 & -0.00285966 \\
\hline & -3 & 0.010824 & 0.112297 & 0.261621 & -0.00223916 & -0.00524012 \\
\hline & -2 & 0.096077 & 0.956645 & -0.00322221 & 0.0577247 & 0.00451714 \\
\hline & -1 & 1.569255 & -0.00663598 & 0.502256 & 0.0219407 & 0.00213071 \\
\hline & 0 & 1960.1 & 2.85307 & 0.0922511 & 0.00637314 & 0.000879331 \\
\hline & 1 & 1.569255 & 0.189722 & 0.0139074 & 0.00204607 & 0.000588102 \\
\hline & 2 & 0.096077 & 0.0212458 & 0.00261023 & 0.00131989 & 0.000979566 \\
\hline & 3 & 0.010824 & 0.0327857 & 0.00115836 & 0.00206073 & 0.00293329 \\
\hline & 4 & 0.001678 & 0.00115417 & 0.00231043 & 0.00615209 & 0.0100231 \\
\hline
\end{tabular}

to instead of $x$ and $t$ in the integrands and integral upper limit to carry out the discrete integration as illustrated below. Then, the obtained discrete integral data points are fitted using the double Fourier series. The functions obtained by fitting can be deemed as the corresponding integral expressions for the mass, momentum, and energy fluxes, respectively. The discrete integration can be described as

$$
\begin{gathered}
Q D_{i, j}^{B W}=\left.\int_{-d}^{\eta_{i, j}} \rho \phi_{x}\left(\theta_{1}, \theta_{2}, z\right)\right|_{\theta_{1}=i \Delta \theta_{1}, \theta_{2}=j \Delta \theta_{2}} \mathrm{~d} z, \\
M D_{i, j}^{B W}=\left.\int_{-d}^{\eta_{i, j}}\left[P\left(\theta_{1}, \theta_{2}, z\right)+\rho \phi_{x}^{2}\left(\theta_{1}, \theta_{2}, z\right)\right]\right|_{\theta_{1}=i \Delta \theta_{1}, \theta_{2}=j \Delta \theta_{2}} \mathrm{~d} z, \\
E D_{i, j}^{B W}=\int_{-d}^{\eta_{i, j}}\left\{P\left(\theta_{1}, \theta_{2}, z\right)+\frac{\rho}{2}\left[\phi_{x}{ }^{2}\left(\theta_{1}, \theta_{2}, z\right)+\phi_{z}{ }^{2}\left(\theta_{1}, \theta_{2}, z\right)\right]+\rho g z\right\} \phi_{x} \\
\times\left.\left(\theta_{1}, \theta_{2}, z\right)\right|_{\theta_{1}=i \Delta \theta_{1}, \theta_{2}=j \Delta \theta_{2}} \mathrm{~d} z,
\end{gathered}
$$

where $\eta_{i, j}=\left.\eta\left(\theta_{1}, \theta_{2}\right)\right|_{\theta_{1}=i \Delta \theta_{1}, \theta_{2}=j \Delta \theta_{2}}, i=0,1, \ldots, I, j=0,1, \ldots, J, I$ and $J$ are the numbers of the discrete points, $\Delta \theta_{1}=4 \pi / I$ and $\Delta \theta_{2}=4 \pi / J$. In the present work, the discrete integrations are calculated with $I=J=20$ to obtain sufficient integral data points for the subsequent fitting. It is worth noting that all the integral quantity expressions for each nonlinear monochromatic wave field based on the HAM solution are similar to those for the bi-chromatic wave field.

To illustrate the calculation and fitting procedure for the discrete integration, consider the bi-chromatic wave case with $\varepsilon_{1}=\varepsilon_{2}=1.008$. As shown in Figs. 5(a)-5(c), the filled circles repre-

\begin{tabular}{|c|c|c|c|c|c|c|}
\hline \multirow[b]{2}{*}{$f_{h, l}$} & & \multicolumn{3}{|c|}{$h$} & \multirow[b]{2}{*}{3} & \multirow[b]{2}{*}{4} \\
\hline & & 0 & 1 & 2 & & \\
\hline \multirow{9}{*}{$l$} & -4 & 0.005637 & 0.0473759 & 0.115674 & 0.0317512 & 0.031869 \\
\hline & -3 & 0.032586 & 0.247497 & 0.0676816 & 0.0948591 & 0.00947649 \\
\hline & -2 & 0.218464 & 0.125411 & 0.27686 & 0.0293963 & 0.0108733 \\
\hline & -1 & 0.087544 & 0.682447 & 0.0771108 & 0.0567149 & 0.00618863 \\
\hline & 0 & 0.477811 & 0.150311 & 0.230699 & 0.0198214 & 0.00241005 \\
\hline & 1 & 0.087544 & 0.450797 & 0.0443137 & 0.00606873 & 0.00138997 \\
\hline & 2 & 0.218464 & 0.0658464 & 0.00897634 & 0.0034472 & 0.00251918 \\
\hline & 3 & 0.032586 & 0.0112076 & 0.00379449 & 0.00557512 & 0.00764964 \\
\hline & 4 & 0.005637 & 0.00397214 & 0.00731952 & 0.016681 & 0.0195476 \\
\hline
\end{tabular}
senting the integral values are obtained by using the above discrete integration. The double Fourier series $F\left(\theta_{1}, \theta_{2}\right)=\sum_{h=0}^{N} \sum_{l=-N}^{N} f_{h, l} \cos \left(h \theta_{1}+l \theta_{2}\right)$ is employed to fit the discrete integral data points to

TABLE V. The coefficients for the fitted function $F_{E}\left(\theta_{1}, \theta_{2}\right)$ for the energy flux of the case BW with $\varepsilon_{1}=\varepsilon_{2}=1.008$. 
TABLE VI. The monochromatic wave parameters $(d=20 \mathrm{~m})$.

\begin{tabular}{lcccccc}
\hline \hline Case & $\varepsilon$ & $\begin{array}{c}\text { Circular frequency } \\
(\mathrm{rad} / \mathrm{s})\end{array}$ & $\begin{array}{c}\text { Wave number } \\
(\mathrm{rad} / \mathrm{m})\end{array}$ & $\begin{array}{c}\text { Wavelength } \\
(\mathrm{m})\end{array}$ & $\begin{array}{c}\text { Phase velocity } \\
(\mathrm{m} / \mathrm{s})\end{array}$ & $\begin{array}{c}\text { Amplitude of primary } \\
\text { waves }(\mathrm{m})\end{array}$ \\
\hline $\mathrm{W} 1$ & 1.002 & 1.98386 & 0.4 & 15.708 & 4.96 & 0.157823 \\
$\mathrm{~W} 2$ & 1.002 & 2.21802 & 0.5 & 12.5664 & 4.44 & 0.126214 \\
\hline \hline
\end{tabular}

obtain the continuous functions for the mass, momentum, and energy fluxes, which are represented by $F_{Q}\left(\theta_{1}, \theta_{2}\right), F_{M}\left(\theta_{1}, \theta_{2}\right)$, and $F_{E}\left(\theta_{1}, \theta_{2}\right)$, respectively. Taking $N=4$, the Fourier coefficients $f_{h, l}$ for the fitted functions for the mass, momentum, and energy fluxes of the bi-chromatic wave case with $\varepsilon_{1}=\varepsilon_{2}=1.008$ were obtained, and shown in Tables III-V, respectively. As clearly shown in Figs. 5(a)-5(c), the curves for the fitted functions agree well with the discrete integral value points, indicating that the corresponding integral quantities can be represented by the fitted functions based on the double Fourier series. Thus, the mean rates of the mass, momentum, and energy fluxes across a vertical section fixed in the bi-chromatic wave field can be obtained as

$$
\begin{aligned}
Q_{F}^{B W} & =\frac{1}{4 \pi^{2}} \int_{0}^{2 \pi} \int_{0}^{2 \pi} F_{Q}\left(\theta_{1}, \theta_{2}\right) \mathrm{d} \theta_{1} \mathrm{~d} \theta_{2}, \\
M_{F}^{B W} & =\frac{1}{4 \pi^{2}} \int_{0}^{2 \pi} \int_{0}^{2 \pi} F_{M}\left(\theta_{1}, \theta_{2}\right) \mathrm{d} \theta_{1} \mathrm{~d} \theta_{2}, \\
E_{F}^{B W} & =\frac{1}{4 \pi^{2}} \int_{0}^{2 \pi} \int_{0}^{2 \pi} F_{E}\left(\theta_{1}, \theta_{2}\right) \mathrm{d} \theta_{1} \mathrm{~d} \theta_{2} .
\end{aligned}
$$

\section{CONSERVATION EQUATIONS}

Baddour and Song ${ }^{19,20}$ proposed the conservation equations based on linear and the secondorder perturbation solutions in terms of the mean rates of the mass, momentum, and energy fluxes of a 2D current-free wave field, a wave-free uniform current field, and a coexisting wave-current field. In their work, the wavelength, wave height, current velocity, and water depth in the combined wave-current field were obtained based on the conservation equations for the mean rates of the mass, momentum, and energy fluxes. Zaman and Baddour ${ }^{21}$ further extended the work of Baddour and Song ${ }^{19,20}$ to a 3D wave-current field in the framework of linear wave theory.

Without loss of generality, consider two 2D, weakly nonlinear, monochromatic wave trains propagating independently in the same direction before encountering. Table VI presents the parameters of the two nonlinear monochromatic wave trains, i.e., cases W1 and W2, respectively, where $\varepsilon=\omega_{i} / \sqrt{g \bar{k}_{i} \tanh \left(\bar{k}_{i} d\right)} ; \omega_{i}$ and $\bar{k}_{i}(i=1,2)$ are the circular frequency and wave number of the corresponding monochromatic waves, respectively, as shown in Fig. 1. It is worth noting that the two

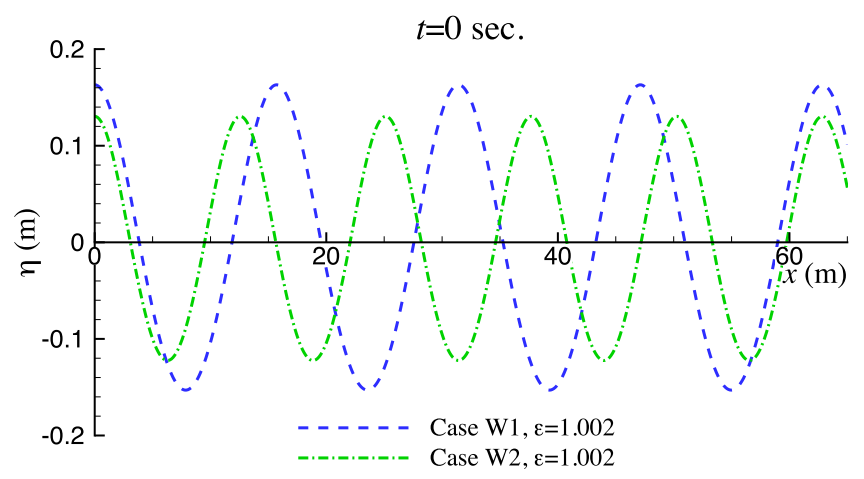

FIG. 6. Wave profile comparison for cases W1 and W2. 
(a)

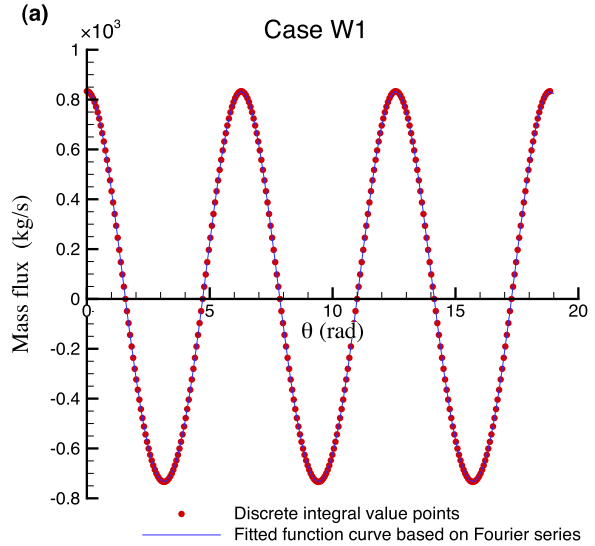

(b)

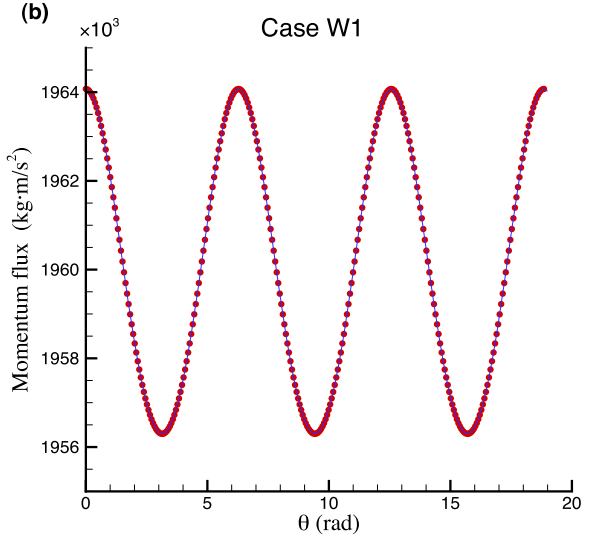

(c)

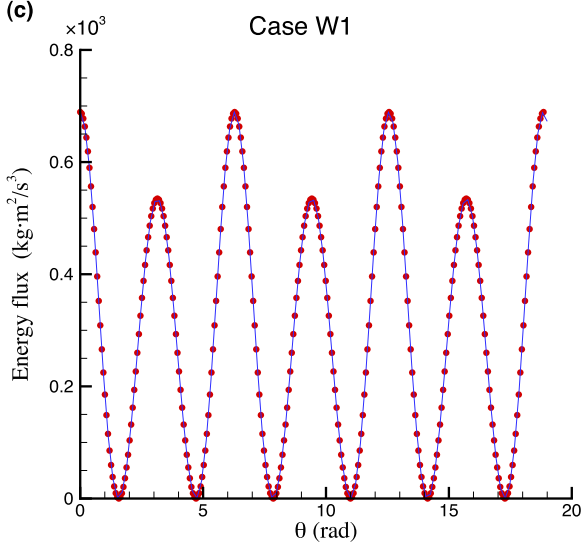

(d)

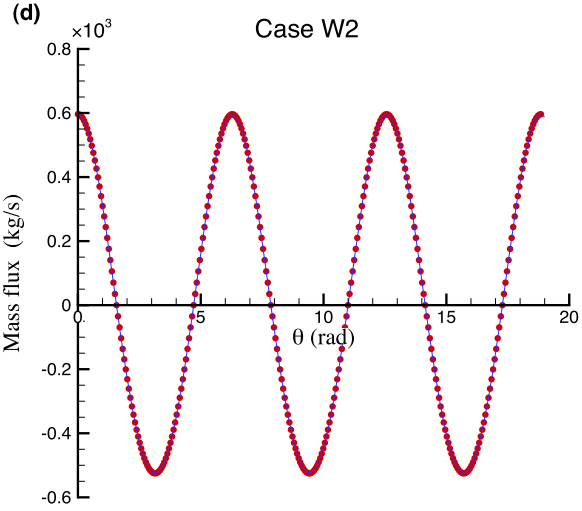

(e)

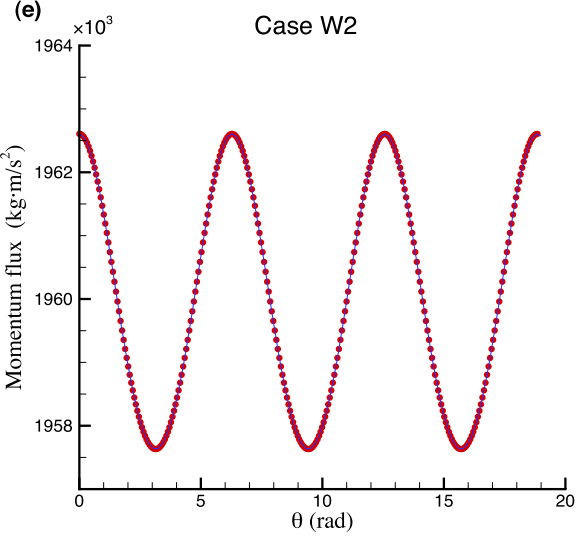

(f)

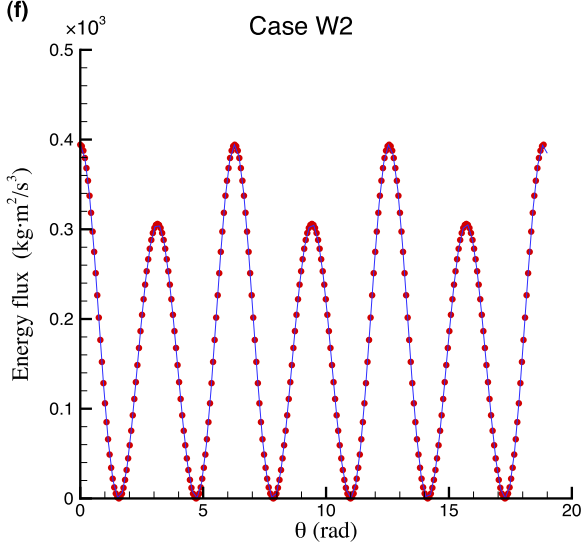

FIG. 7. The comparison of the discrete integral value points and fitted function curves for (a) and (d): mass flux; (b) and (e): momentum flux; (c) and (f): energy flux of cases W1 and W2.

monochromatic wave cases in the paper are indeed weakly nonlinear. This is due to the assumption that the bi-chromatic wave system is obtained by the nonlinear interaction of the two monochromatic wave trains with wave frequencies and water depth unchanged. However, the interaction of these two weakly nonlinear monochromatic wave cases leads to a sufficiently strong nonlinear bi-chromatic wave system at a higher conservation level of mass, momentum, and energy fluxes, as discussed in the following. In fact, Liao and Cheung ${ }^{13}$ and Tao et al. ${ }^{14}$ presented accurate HAM solutions for strongly nonlinear, monochromatic progressive waves in deep and finite water depth, respectively, in which the maximum wave steepness $(H / L)$ is close to the limiting wave steepness 
TABLE VII. The mean rates of the mass, momentum, and energy fluxes of cases $\mathrm{W} 1$ and $\mathrm{W} 2$.

\begin{tabular}{lccc}
\hline \hline Case & Mass flux $(\mathrm{kg} / \mathrm{s})$ & $\begin{array}{c}\text { Momentum } \\
\text { flux }\left(\mathrm{kg} \mathrm{m} / \mathrm{s}^{2}\right)\end{array}$ & $\begin{array}{c}\text { Energy } \\
\left.\text { flux }(\mathrm{kg} \mathrm{m})^{2} / \mathrm{s}^{3}\right)\end{array}$ \\
\hline W1 & $0.0246829 \times 10^{3}$ & $1960.06 \times 10^{3}$ & $0.304792 \times 10^{3}$ \\
W2 & $0.0176491 \times 10^{3}$ & $1960.04 \times 10^{3}$ & $0.174348 \times 10^{3}$ \\
\hline \hline
\end{tabular}

$(0.142 \tanh k d)$. All the HAM solutions for cases W1 and W2 are obtained with a total averaged residual error at least $10^{-6}$, which is defined in the Appendix and used to describe the accuracy of the homotopy series solution. Fig. 6 shows the wave profiles for cases $\mathrm{W} 1$ and $\mathrm{W} 2$ at $t=0$. Fig. 7 shows the comparison of the discrete integral value points and the fitted function curves for the mass, momentum, and energy fluxes for cases W1 and W2, respectively. It is clearly seen that the curves for the fitted functions agree well with the corresponding discrete integral value points. By means of the obtained fitted functions, the mean rates of the mass, momentum, and energy fluxes across a fixed section, which are denoted by $Q_{F}^{W 1}, M_{F}^{W 1}$, and $E_{F}^{W 1}$ for case W1 and by $Q_{F}^{W 2}, M_{F}^{W 2}$, and $E_{F}^{W 2}$ for case W2, respectively, are calculated and presented in Table VII. The conservation equations for wave-wave interaction can be summarized as

$$
\begin{gathered}
Q_{F}^{W 1}+Q_{F}^{W 2}=Q_{F}^{B W}, \\
M_{F}^{W 1}+M_{F}^{W 2}=M_{F}^{B W}, \\
E_{F}^{W 1}+E_{F}^{W 2}=E_{F}^{B W} .
\end{gathered}
$$

It is noted that the approximate solutions for the conservation equations of the mass, momentum, and energy fluxes were obtained by Baddour and Song ${ }^{19,20}$ based on the low-order perturbation solutions for the current-free wave field and combined wave-current field. Due to the nonlinear feature of the interaction process, it is difficult to obtain the exact solutions for the bi-chromatic wave field, which is obtained via the interaction of the two nonlinear monochromatic wave trains, by solving the conservation Eqs. (27)-(29). Thus, in this study, the standard deviation

$$
S_{d}=\sqrt{\frac{\left(r_{Q}-1\right)^{2}+\left(r_{M}-1\right)^{2}+\left(r_{E}-1\right)^{2}}{3}}
$$

is defined to illustrate the deviation from the conservation state $\left(S_{d}=0\right)$ of the mean rates of the mass, momentum, and energy fluxes before and after the interaction of the two nonlinear monochromatic wave trains, where

$$
r_{Q}=\frac{Q_{F}^{B W}}{Q_{F}^{W 1}+Q_{F}^{W 2}}, \quad r_{M}=\frac{M_{F}^{B W}}{M_{F}^{W 1}+M_{F}^{W 2}}, \quad r_{E}=\frac{E_{F}^{B W}}{E_{F}^{W 1}+E_{F}^{W 2}} .
$$

Using the standard deviation $S_{d}$, it is not difficult to obtain a state evaluating the deviation from the conservation state after the interaction of the two monochromatic wave trains.

\section{RESULTS AND DISCUSSION}

\section{A. Analyses based on $\varepsilon_{1}=\varepsilon_{2}$}

It is assumed that the nonlinear monochromatic wave case $\mathrm{W} 1$ with a higher phase velocity $(4.96 \mathrm{~m} / \mathrm{s})$ will catch up to the case W2 with a lower phase velocity $(4.44 \mathrm{~m} / \mathrm{s})$ and interact thoroughly, which results in the formation of a steady-state bi-chromatic wave field. The frequencies of the primary waves and water depth are assumed to be invariant before and after the interaction. As abovementioned, it is quite difficult to obtain the exact solutions for the conservation equations due to the nonlinear feature. The practice of this paper is to search the solutions for the conservation equations, which are applied to assess the deviation $S_{d}$ from the conservation state $\left(S_{d}=0\right)$. First, 
TABLE VIII. The bi-chromatic wave parameters in the case of $\varepsilon_{1}=\varepsilon_{2}(d=20 \mathrm{~m})$.

\begin{tabular}{lcccccccc}
\hline \hline$\varepsilon_{1}$ & $\varepsilon_{2}$ & $\omega_{1}(\mathrm{rad} / \mathrm{s})$ & $\omega_{2}(\mathrm{rad} / \mathrm{s})$ & $S_{d}$ & $L_{1}(\mathrm{~m})$ & $L_{2}(\mathrm{~m})$ & $a_{1,0}(\mathrm{~m})$ & $a_{0,1}(\mathrm{~m})$ \\
\hline 1.001 & 1.001 & 1.98386 & 2.21802 & 0.682 & 15.6766 & 12.5413 & 0.0487901 & 0.0660159 \\
1.002 & 1.002 & 1.98386 & 2.21802 & 0.555 & 15.7079 & 12.5664 & 0.0678623 & 0.0918262 \\
1.003 & 1.003 & 1.98386 & 2.21802 & 0.436 & 15.7393 & 12.5915 & 0.0814162 & 0.11022 \\
1.004 & 1.004 & 1.98386 & 2.21802 & 0.327 & 15.7707 & 12.6166 & 0.0917175 & 0.124255 \\
1.005 & 1.005 & 1.98386 & 2.21802 & 0.229 & 15.8021 & 12.6417 & 0.0996403 & 0.135117 \\
1.006 & 1.006 & 1.98386 & 2.21802 & 0.140 & 15.8336 & 12.6669 & 0.105687 & 0.143485 \\
1.007 & 1.007 & 1.98386 & 2.21802 & 0.061 & 15.8651 & 12.6921 & 0.110286 & 0.149906 \\
1.008 & 1.008 & 1.98386 & 2.21802 & 0.013 & 15.8966 & 12.7173 & 0.113925 & 0.154952 \\
1.009 & 1.009 & 1.98386 & 2.21802 & 0.082 & 15.9282 & 12.7426 & 0.117177 & 0.159348 \\
1.01 & 1.01 & 1.98386 & 2.21802 & 0.157 & 15.9598 & 12.7679 & 0.120801 & 0.164024 \\
\hline \hline
\end{tabular}

a set of $\varepsilon_{1}\left(=\varepsilon_{2}\right)$ for the bi-chromatic wave field is utilized to investigate the deviation from the conservation state by means of the standard deviation $S_{d}$. Table VIII shows the bi-chromatic wave parameters for the conservation study. For the bi-chromatic wave field, for simplicity, it is easy to assume that $\varepsilon_{1}=\varepsilon_{2}$ with the same value (1.002) as that of the nonlinear monochromatic wave fields. As shown in Table VIII, for $\varepsilon_{1}=\varepsilon_{2}=1.002, S_{d}=0.555$ can be obtained based on the HAM solutions. However, for $\varepsilon_{1}=\varepsilon_{2}=1.001, S_{d}$ tends to approach a much higher value (0.682). This indicates that a smaller value for $\varepsilon_{1}\left(=\varepsilon_{2}\right)$ leads to a larger discrepancy between the mean rates of the mass, momentum, and energy fluxes before and after the interaction. For $\varepsilon_{1}\left(=\varepsilon_{2}\right)$ from 1.003 to 1.01 with an increment of $0.001, S_{d}$ approaches a relatively smaller value $(0.013)$ at $\varepsilon_{1}=\varepsilon_{2}=1.008$, indicating that the case $\left(\varepsilon_{1}=\varepsilon_{2}=1.008\right)$ is much closer to the conservation state of the mass, momentum, and energy fluxes than other cases in Table VIII.

Fig. 8 shows the wave profile comparison for the cases BW with $\varepsilon_{1}=\varepsilon_{2}=1.006,1.008$, and 1.01 at $t=0$. It can be clearly seen that the largest wave crest at $x=0$ becomes higher and higher as $\varepsilon_{1}\left(=\varepsilon_{2}\right)$ increases, whilst the largest wave trough next to $x=0$ becomes lower and lower. This means that the largest wave height in the wave profile tends to increase notably as $\varepsilon_{1}$ increases, although the increment in $\varepsilon_{1}$ is very small. However, the profiles of the wave crest and trough around $x=30 \mathrm{~m}$ appear to be invariant.

To investigate the frequency content of the bi-chromatic wave system, the time series of the wave elevation obtained from the HAM solutions are analyzed by FFT. Figs. 9(a)-9(c) show the amplitude spectra of the bi-chromatic wave system with $\varepsilon_{1}=\varepsilon_{2}=1.006,1.008$, and 1.01 . As shown in Figs. 9(a)-9(c), two dominant large amplitudes (at $f_{1} \approx 0.314367 \mathrm{~Hz}$ and $f_{2} \approx$ $0.352342 \mathrm{~Hz}$ ) can be clearly seen and high-order nonlinear components of each bi-chromatic wave case are rather prominent. As $\varepsilon_{1}\left(=\varepsilon_{2}\right)$ increases from 1.006 to 1.01 , the nonlinearity becomes stronger and stronger and some higher-order wave components, e.g., the third order $\left(3 f_{1}, 3 f_{2}\right.$, etc.), become increasingly significant. Table IX presents the amplitudes of the wave components from the

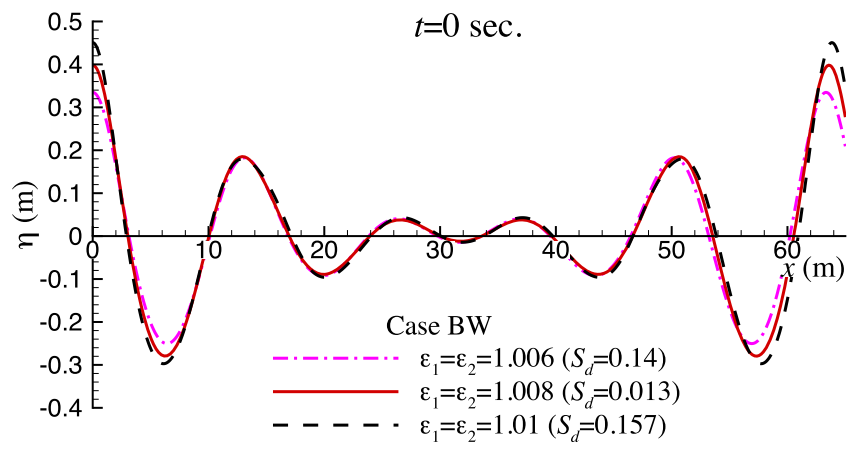

FIG. 8. Wave profile comparison between the cases BW with $\varepsilon_{1}=\varepsilon_{2}=1.006,1.008$, and 1.01. 
(a)

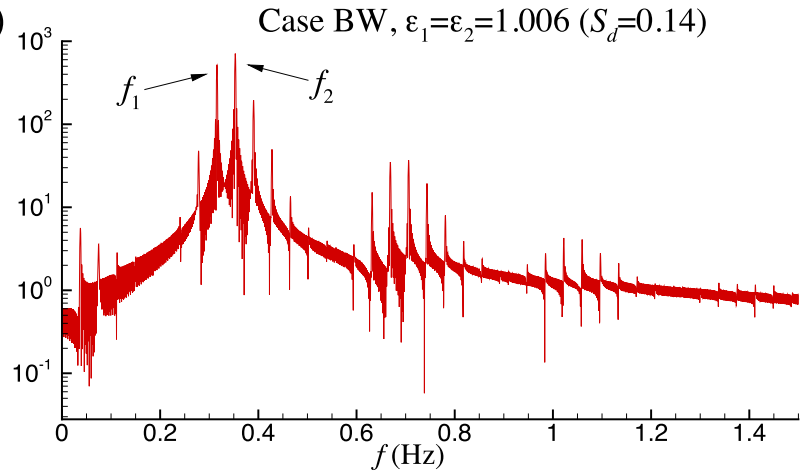

(b)

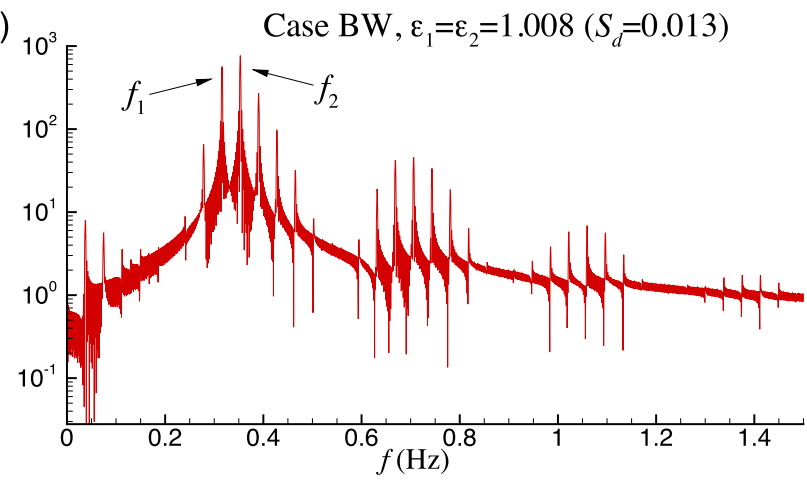

(c)

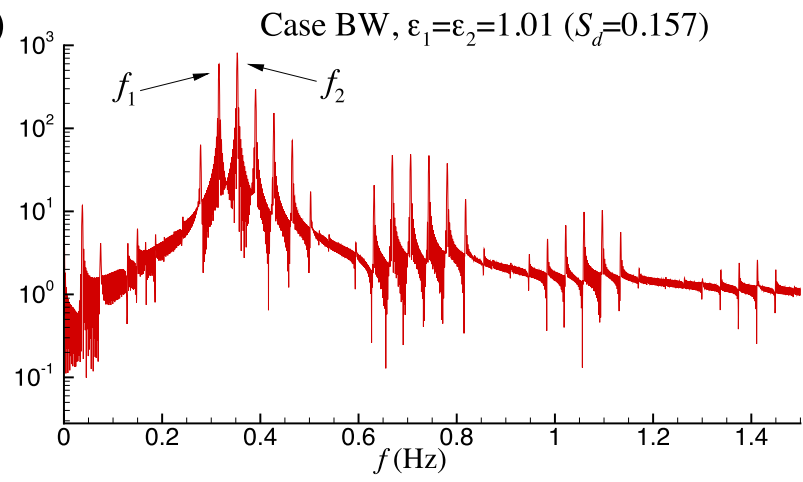

FIG. 9. Amplitude spectra for the cases BW with $\varepsilon_{1}=\varepsilon_{2}=1.006,1.008$, and 1.01.

first order to the third order of the cases BW with $\varepsilon_{1}=\varepsilon_{2}=1.006,1.008$, and 1.01, respectively. It can be clearly observed that the amplitudes of the wave components from the first order to the third order between these cases have remarkable difference. For example, the value for $a\left(f_{1}\right)$ in the case of $\varepsilon_{1}=\varepsilon_{2}=1.008\left(S_{d}=0.013\right)$ is $0.113925 \mathrm{~m}$ which is $7.5 \%$ greater than $0.105687 \mathrm{~m}$ in the case of $\varepsilon_{1}=\varepsilon_{2}=1.006\left(S_{d}=0.140\right)$, and $6.1 \%$ less than $0.120801 \mathrm{~m}$ in the case of $\varepsilon_{1}=\varepsilon_{2}=$ $1.01\left(S_{d}=0.157\right)$. In fact, these different amplitudes indeed lead to the difference of the mean rates of the mass, momentum, and energy fluxes between these three cases, which can be assessed by the standard deviation $\left(S_{d}\right)$ values from the conservation state.

\section{B. Analyses based on $\varepsilon_{1} \neq \varepsilon_{2}$}

As shown in Table VIII, it can be seen that $S_{d}$ approaches a relatively smaller value (0.013) in the case of $\varepsilon_{1}=\varepsilon_{2}=1.008$, which represents that the case $\left(\varepsilon_{1}=\varepsilon_{2}=1.008\right)$ is much closer to the 
TABLE IX. Amplitudes of the wave components from the first order to the third order of the cases BW with $\varepsilon_{1}=\varepsilon_{2}=1.006$, $1.008,1.01 ; \varepsilon_{1}=1.007, \varepsilon_{2}=1.008$ and $\varepsilon_{1}=1.0065, \varepsilon_{2}=1.008$.

\begin{tabular}{lccccc}
\hline \hline & \multicolumn{5}{c}{$\varepsilon_{1}\left(=\varepsilon_{2}\right)$} \\
Amplitude $(\mathrm{m})$ & 1.006 & 1.008 & 1.01 & $\varepsilon_{1}=1.007, \varepsilon_{2}=1.008$ & $\varepsilon_{1}=1.0065, \varepsilon_{2}=1.008$ \\
\cline { 2 - 5 }$a\left(f_{1}\right)$ & 0.105687 & 0.113925 & 0.120801 & 0.129123 & 0.138147 \\
$a\left(f_{2}\right)$ & 0.143485 & 0.154952 & 0.164024 & 0.144214 & 0.137890 \\
$a\left(2 f_{1}\right)$ & 0.002907 & 0.003627 & 0.003950 & 0.004442 & 0.004922 \\
$a\left(2 f_{2}\right)$ & 0.007326 & 0.009165 & 0.009710 & 0.008641 & 0.008029 \\
$a\left(f_{1}+f_{2}\right)$ & 0.007133 & 0.008508 & 0.009574 & 0.008875 & 0.009034 \\
$a\left(f_{1}-f_{2}\right)$ & 0.001206 & 0.001675 & 0.002460 & 0.001493 & 0.001472 \\
$a\left(3 f_{1}\right)$ & 0.000139 & 0.000212 & 0.000263 & 0.000284 & 0.000319 \\
$a\left(3 f_{2}\right)$ & 0.000738 & 0.001256 & 0.001849 & 0.000974 & 0.000710 \\
$a\left(2 f_{1}+f_{2}\right)$ & 0.000432 & 0.000604 & 0.000753 & 0.000668 & 0.000965 \\
$a\left(f_{1}+2 f_{2}\right)$ & 0.000718 & 0.000992 & 0.001171 & 0.000985 & 0.016353 \\
$a\left(2 f_{1}-f_{2}\right)$ & 0.009398 & 0.013054 & 0.012437 & 0.015433 & 0.043462 \\
$a\left(f_{1}-2 f_{2}\right)$ & 0.038920 & 0.054585 & 0.060127 & 0.048538 & \\
\hline \hline
\end{tabular}

conservation state $\left(S_{d}=0\right)$ of the mean rates of the mass, momentum, and energy fluxes before and after the interaction than other cases in Table VIII. Obviously, the above analysis is based on the assumption that $\varepsilon_{1}$ is equal to $\varepsilon_{2}$ for the steady-state bi-chromatic wave field after the interaction. However, it is essential to consider $\varepsilon_{1} \neq \varepsilon_{2}$ for the bi-chromatic wave field. Table $\mathrm{X}$ presents the values for $S_{d}$ for the matrix of $\varepsilon_{1}$ and $\varepsilon_{2}$ ranging from 1.005 to 1.01 with an increment of 0.001 . As shown in Table X, the minimum values for $S_{d}$ in each row $(0.02,0.010,0.009,0.013,0.027,0.034)$ arises in the same column in which $\varepsilon_{2}=1.008$. For the column with $\varepsilon_{2}=1.008$, it is noted that the minimum value for $S_{d}$ is 0.009 which is calculated in the case of $\varepsilon_{1}=1.007$ and $\varepsilon_{2}=1.008$. This indicates that $\varepsilon_{1} \neq \varepsilon_{2}$ can yield a lower value for $S_{d}$ compared to the cases in Table VIII which are based on the assumption that $\varepsilon_{1}=\varepsilon_{2}$, e.g., there is a slight difference $(0.004)$ for $S_{d}$ in the case of $\varepsilon_{1}=\varepsilon_{2}=1.008$ and $\varepsilon_{1}=1.007, \varepsilon_{2}=1.008$. It is worth noting that all the values for $S_{d}$ presented in Table $\mathrm{X}$ are calculated based on the HAM solutions with a total averaged residual error at least $10^{-6}$ for each steady wave field.

Table XI further presents the values for $S_{d}$ for the matrix of $\varepsilon_{1}$ and $\varepsilon_{2}$ around $\varepsilon_{1}=1.007$ and $\varepsilon_{2}=1.008$ with a smaller increment of 0.0005 . As shown in Table XI, similar to the tendency in Table X, the minimum values for $S_{d}$ in each row arise in the column with $\varepsilon_{2}=1.008$. It is clear that compared to the case of $\varepsilon_{1}=1.007$ and $\varepsilon_{2}=1.008$, a smaller value $(0.007)$ for $S_{d}$ can be obtained when $\varepsilon_{1}=1.0065$ and $\varepsilon_{2}=1.008$. This indicates that it is possible to obtain much smaller values for $S_{d}$ by subdividing around $\varepsilon_{1}=1.007$ and $\varepsilon_{2}=1.008$ with a smaller increment. However, in this paper, the case BW with $\varepsilon_{1}=1.0065, \varepsilon_{2}=1.008$, and $S_{d}=0.007$ is supposed to be highly close to the conservation state, thus it is not essential to seek smaller values for $S_{d}$.

TABLE X. The standard deviation $S_{d}$ for the matrix of $\varepsilon_{1}$ and $\varepsilon_{2}$ with an increment of 0.001 .

\begin{tabular}{cccccccc}
\hline \hline & & \multicolumn{6}{c}{$\varepsilon_{2}$} \\
\cline { 3 - 7 }$S_{d}$ & & 1.005 & 1.006 & 1.007 & 1.008 & 1.009 & 1.01 \\
\hline \multirow{4}{*}{$n^{*}$} & 1.005 & 0.229 & 0.150 & 0.070 & 0.020 & 0.098 & $\ldots$ \\
& 1.006 & 0.209 & 0.140 & 0.069 & 0.010 & 0.094 & 0.172 \\
& 1.007 & 0.193 & 0.132 & 0.061 & 0.009 & 0.087 & 0.167 \\
& 1.008 & 0.185 & 0.125 & 0.066 & 0.013 & 0.073 & 0.151 \\
& 1.009 & 0.105 & 0.121 & 0.067 & 0.027 & 0.082 & 0.130 \\
& 1.01 & $\ldots$ & 0.138 & 0.082 & 0.034 & 0.067 & 0.157 \\
\hline \hline
\end{tabular}


TABLE XI. The standard deviation $S_{d}$ for the matrix of $\varepsilon_{1}$ and $\varepsilon_{2}$ with an increment of 0.0005 .

\begin{tabular}{lllllll}
\hline \hline & & \multicolumn{5}{c}{$\varepsilon_{2}$} \\
\cline { 3 - 7 }$S_{d}$ & & 1.007 & 1.0075 & 1.008 & 1.0085 & 1.009 \\
\hline \multirow{4}{*}{$\varepsilon_{1}$} & 1.006 & 0.069 & 0.031 & 0.010 & 0.049 & 0.094 \\
& 1.0065 & 0.068 & 0.031 & 0.007 & 0.046 & 0.086 \\
& 1.007 & 0.061 & 0.032 & 0.009 & 0.044 & 0.087 \\
& 1.0075 & 0.066 & 0.034 & 0.012 & 0.041 & 0.078 \\
\hline \hline
\end{tabular}

Fig. 10 shows the wave profiles at $t=0$ for the cases BW with $\varepsilon_{1}=1.0065, \varepsilon_{2}=1.008$, $\varepsilon_{1}=1.007, \varepsilon_{2}=1.008$, and $\varepsilon_{1}=\varepsilon_{2}=1.008$. In contrast to the characteristics in Fig. 8, it can be seen in Fig. 10 that for the three bi-chromatic wave cases, no significant difference exists for the profile of the largest wave crest at $x=0$ and wave trough next to $x=0$, whilst slight difference arises between the wave crest and wave trough around $x=30$. This indicates that the slight difference for $S_{d}$ between the three bi-chromatic wave cases does not lead to remarkable influence on the wave profile. Fig. 11 shows the amplitude spectra of the bi-chromatic wave cases BW with $\varepsilon_{1}=1.0065, \varepsilon_{2}=1.008$ and $\varepsilon_{1}=1.007, \varepsilon_{2}=1.008$. It is seen again that high-order nonlinear wave components are quite evident. It is also noted that for the case BW with $\varepsilon_{1}=1.007, \varepsilon_{2}=1.008$, the amplitude of the primary wave $f_{2}$ appears to be slightly greater than that of the primary wave $f_{1}$; whilst for the case BW with $\varepsilon_{1}=1.0065, \varepsilon_{2}=1.008$, the amplitude of the primary wave $f_{2}$ appears to be identical to that of the primary wave $f_{1}$. To further see the amplitudes of various order wave components, the amplitudes of the wave components from the first order to the third order of the cases BW with $\varepsilon_{1}=1.007, \varepsilon_{2}=1.008$ and $\varepsilon_{1}=1.0065, \varepsilon_{2}=1.008$ are also presented in Table IX. It is observed in Table IX that the amplitudes of various order wave components of the cases BW with $\varepsilon_{1}=\varepsilon_{2}=1.008, \varepsilon_{1}=1.007, \varepsilon_{2}=1.008$ and $\varepsilon_{1}=1.0065, \varepsilon_{2}=1.008$ appear to be evidently different. It is these differences that produce the discrepancy of the mean rates of the mass, momentum, and energy fluxes between these three cases. On the other hand, for the case BW with $\varepsilon_{1}=1.0065$ and $\varepsilon_{2}=1.008$, which leads to a smaller value $(0.007)$ for $S_{d}$, the ratio of the primary wave amplitudes $\left(a\left(f_{1}\right) / a\left(f_{2}\right) \approx 1.002\right)$ tends to approach 1 compared to the cases BW with $\varepsilon_{1}=1.007, \varepsilon_{2}=1.008\left(a\left(f_{1}\right) / a\left(f_{2}\right) \approx 0.895\right)$, and $\varepsilon_{1}=\varepsilon_{2}=1.008\left(a\left(f_{1}\right) / a\left(f_{2}\right) \approx 0.735\right)$. This means that the energy of the primary waves tends to balance each other for the case BW which is much closer to the conservation state after the interaction.

Fig. 12 shows the amplitudes of the primary waves of the monochromatic wave cases W1, W2, as well as the bi-chromatic wave case BW with $\varepsilon_{1}=1.0065$ and $\varepsilon_{2}=1.008$. As shown in Fig. 12, the amplitudes of the primary waves of each nonlinear monochromatic wave train before the interaction are $0.157823 \mathrm{~m}$ and $0.126214 \mathrm{~m}$, respectively, while the corresponding amplitudes of the

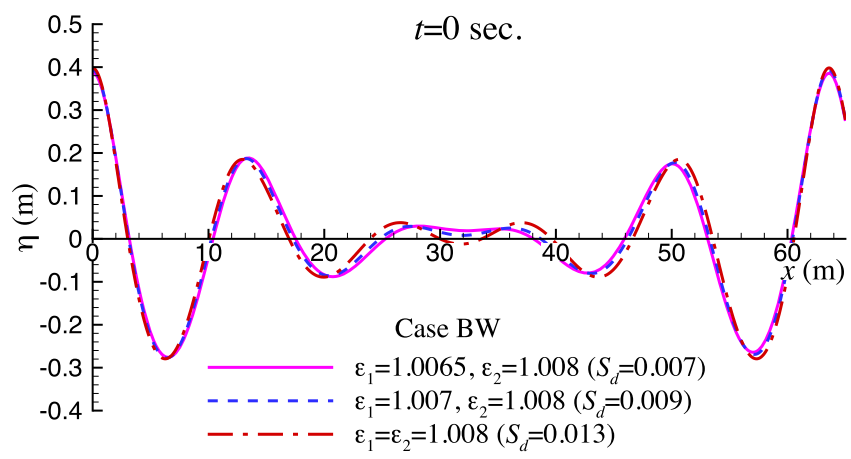

FIG. 10. Wave profile comparison for the cases BW with $\varepsilon_{1}=1.0065, \varepsilon_{2}=1.008 ; \varepsilon_{1}=1.007, \varepsilon_{2}=1.008$, and $\varepsilon_{1}=\varepsilon_{2}=$ 1.008 . 
(a)

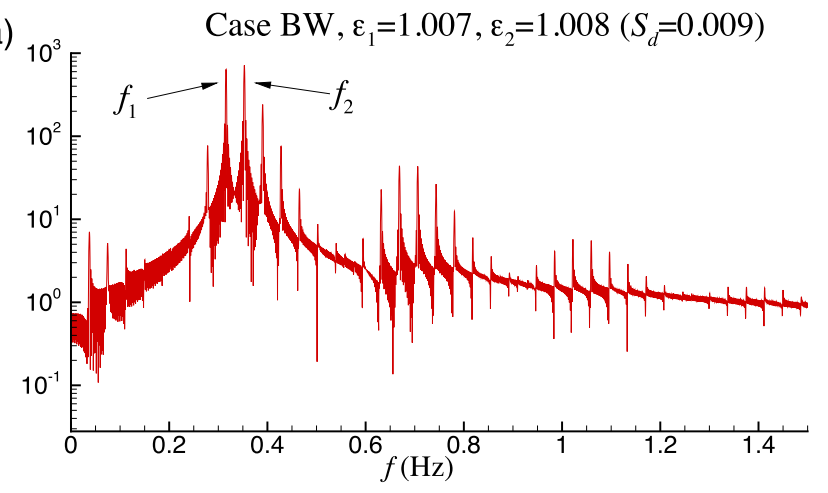

(b)

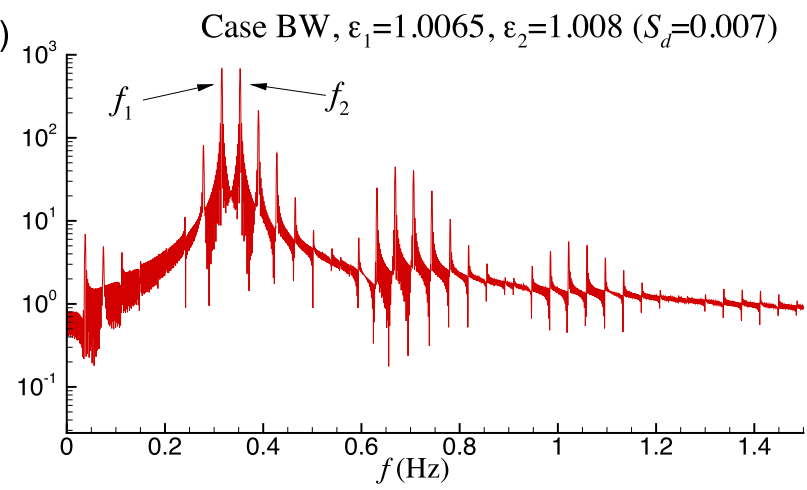

FIG. 11. Amplitude spectra for the cases BW with $\varepsilon_{1}=1.007, \varepsilon_{2}=1.008$ and $\varepsilon_{1}=1.0065, \varepsilon_{2}=1.008$.

primary waves of the bi-chromatic wave field with $\varepsilon_{1}=1.0065$ and $\varepsilon_{2}=1.008$ are $0.138147 \mathrm{~m}$ and $0.13789 \mathrm{~m}$, respectively. The amplitude of the primary wave with a lower frequency $\left(\omega_{1}=\right.$ $1.98386 \mathrm{rad} / \mathrm{s}$ ) drops from $0.157823 \mathrm{~m}$ to $0.138147 \mathrm{~m}$ with a decrement approximately $18.4 \%$, while the one with a higher frequency $\left(\omega_{2}=2.21802 \mathrm{rad} / \mathrm{s}\right)$ increases from $0.126214 \mathrm{~m}$ to $0.13789 \mathrm{~m}$ with an increment approximately $14.3 \%$. It is clear that the amplitude of the primary

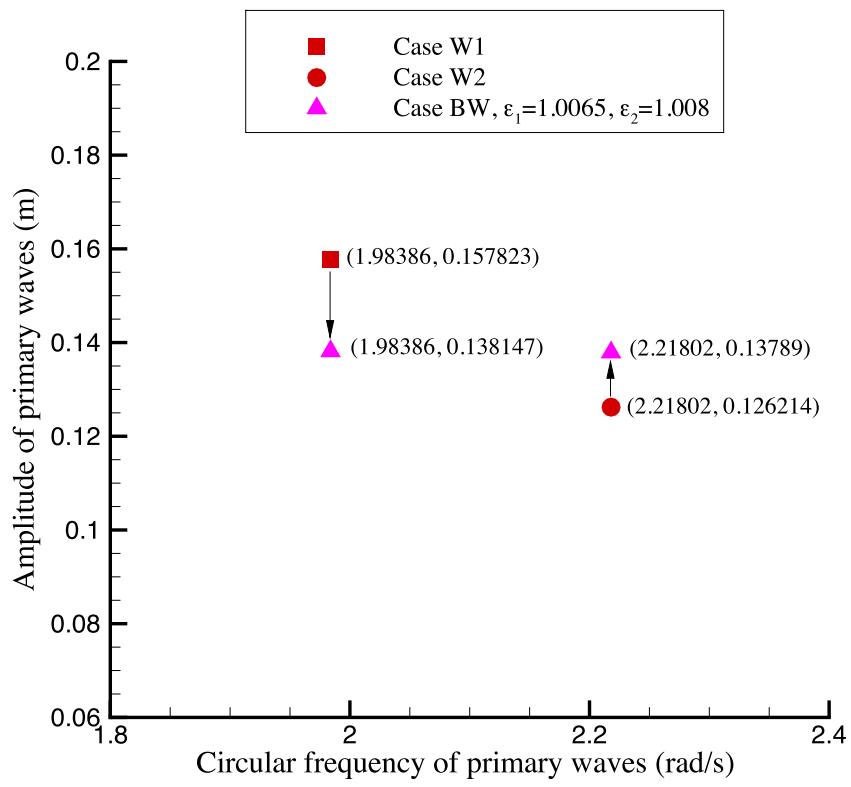

FIG. 12. Amplitudes of the primary waves of cases W1, W2, and the case BW with $\varepsilon_{1}=1.0065, \varepsilon_{2}=1.008$. 


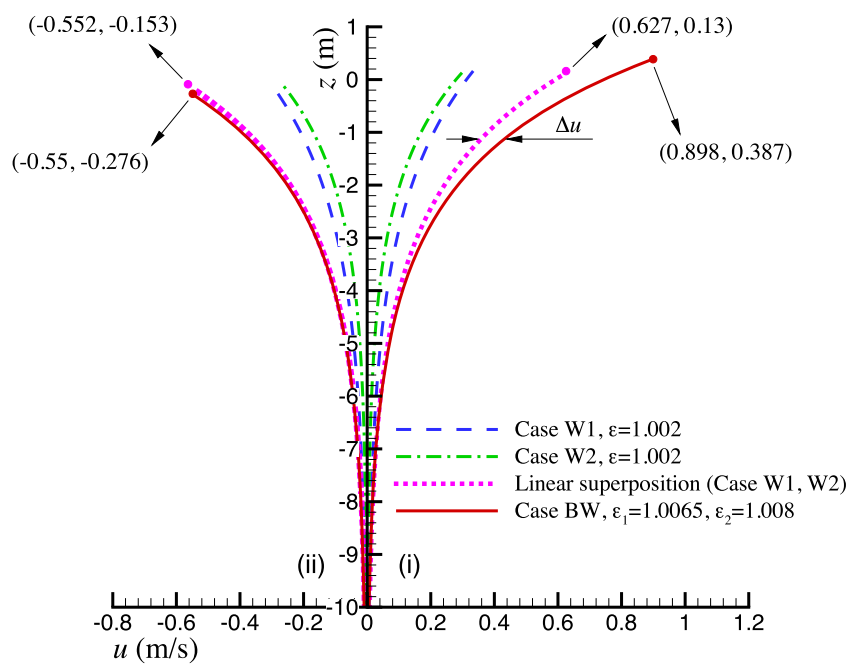

FIG. 13. Water particle horizontal velocity profiles at $t=0$ : (i) under the wave crest at $x=0$; (ii) under the wave trough next to $x=0$.

wave with a lower frequency tends to decrease; while the one with a higher frequency tends to increase in terms of $S_{d}=0.007$ of the mean rates of the mass, momentum, and energy fluxes before and after the interaction. This demonstrates the energy transfer from the primary wave with a lower frequency to that with a higher frequency during the interaction.

Fig. 13 shows the horizontal velocity profiles underneath the wave crest and wave trough for the monochromatic wave cases W1, W2, and the bi-chromatic wave case with $\varepsilon_{1}=1.0065$ and $\varepsilon_{2}=1.008$ which is much closer to the conservation state. The profiles of the linear superposition of the velocity profiles for cases W1 and W2 are also presented in Fig. 13. As shown in Fig. 13, for the bi-chromatic wave case, the largest horizontal velocity of the water particle on the largest wave trough (next to $x=0$ ) is approximately $0.55 \mathrm{~m} / \mathrm{s}$ which is almost identical to the linear superposition value $(0.552 \mathrm{~m} / \mathrm{s})$ of the largest horizontal velocities on the wave trough of the two monochromatic wave cases $\mathrm{W} 1$ and W2; whilst the largest horizontal velocity of the water particle on the largest wave crest (at $x=0$ ) of the bi-chromatic wave case is approximately $0.898 \mathrm{~m} / \mathrm{s}$ which is 1.43 times the linear superposition value $(0.627 \mathrm{~m} / \mathrm{s})$ of the largest horizontal velocities on the wave crest of the two monochromatic wave cases W1 and W2. It is also noted that for the case BW with $\varepsilon_{1}=1.0065, \varepsilon_{2}=1.008$, and the linear superposition of cases $\mathrm{W} 1$ and $\mathrm{W} 2$, the differences between the velocity profiles under the wave crests $(\Delta u)$ gradually diminish as water depth deepens and tend to coincide with each other when the depth is deeper than $-d / 5$ (i.e., $-4 \mathrm{~m}$ ); while the velocity profiles under the wave trough appear to coincide along the whole water depth. This evidently indicates that the nonlinear interaction between the two monochromatic waves leads to significant increases in the horizontal velocity of the water particles under the largest wave crest compared to the corresponding linear superposition values, especially close to the free surface.

\section{CONCLUSIONS}

Nonlinear progressive bi-chromatic waves in water of finite depth are studied by using the homotopy analysis method. The equations for the mass, momentum, and energy fluxes based on accurate high-order homotopy series solutions are derived using the discrete integrations and Fourier series-based fittings. The relationship between the steady-state bi-chromatic wave field and the two nonlinear monochromatic wave trains is established in terms of the conservation equations for the mean rates of the mass, momentum, and energy fluxes before and after the interaction. The parametric analysis on $\varepsilon_{1}$ and $\varepsilon_{2}$ of the bi-chromatic wave field is performed to obtain sufficiently small values for the standard deviation $S_{d}$, which is applied to describe the deviation from the 
conservation state $\left(S_{d}=0\right)$ before and after the interaction of the two nonlinear monochromatic wave trains. The following conclusions are drawn from this study.

1. The discrete integration and the fitting based on the Fourier series can provide accurate expressions for the mass, momentum, and energy fluxes of a monochromatic wave field as well as a steady-state bi-chromatic wave field.

2. Under the assumption either $\varepsilon_{1}=\varepsilon_{2}$ or $\varepsilon_{1} \neq \varepsilon_{2}$, some cases $\left(S_{d} \leq 0.013\right)$ are found to be very close to the conservation state $\left(S_{d}=0\right)$ of the mean rates of the mass, momentum, and energy fluxes before and after the interaction.

3. The amplitude of the primary wave with a lower frequency tends to decrease, and the one with a higher frequency tends to increase based on the conservation analysis on the mean rates of the mass, momentum, and energy fluxes before and after the interaction.

4. The energy of the primary waves of the bi-chromatic wave case BW which is much closer to the conservation state $\left(S_{d}=0\right)$ after the interaction, tends to balance each other.

5. The nonlinear interaction between the two monochromatic waves is found to lead to significant increases in the horizontal velocity of the water particles under the largest wave crest, especially close to the free surface.

\section{ACKNOWLEDGMENTS}

The authors would like to express their gratitude to the National Natural Science Foundation of China (Grant Nos. 51239007 and 51209136) and Newton Research Collaboration Programme Award, The Royal Academy of Engineering UK for financial support.

\section{APPENDIX: SOLUTION PROCEDURE BY HAM}

\section{Zeroth-order deformation equation}

In the framework of HAM, there is great freedom to choose the linear auxiliary operator. According to the linear part of the nonlinear boundary conditions (10) and (11), two linear auxiliary operators are chosen as

$$
\begin{gathered}
\mathrm{L}_{1}[(\cdot)]=(\cdot), \\
\mathrm{L}_{2}[\phi]=\bar{\omega}_{1}^{2} \frac{\partial^{2} \phi}{\partial \theta_{1}{ }^{2}}+2 \bar{\omega}_{1} \bar{\omega}_{2} \frac{\partial^{2} \phi}{\partial \theta_{1} \partial \theta_{2}}+\bar{\omega}_{2}^{2} \frac{\partial^{2} \phi}{\partial \theta_{2}{ }^{2}}+g \frac{\partial \phi}{\partial z}
\end{gathered}
$$

where

$$
\bar{\omega}_{i}=\sqrt{g k_{i} \tanh \left(k_{i} d\right)}(i=1,2) .
$$

Based on the nonlinear boundary conditions, two nonlinear operators can be defined as

$$
\begin{gathered}
\mathrm{N}_{1}[\eta, \phi]=\eta-\frac{1}{g}\left(\omega_{1} \frac{\partial \phi}{\partial \theta_{1}}+\omega_{2} \frac{\partial \phi}{\partial \theta_{2}}-f\right), \\
\mathrm{N}_{2}[\phi]=\omega_{1}^{2} \frac{\partial^{2} \phi}{\partial \theta_{1}^{2}}+2 \omega_{1} \omega_{2} \frac{\partial^{2} \phi}{\partial \theta_{1} \partial \theta_{2}}+\omega_{2}^{2} \frac{\partial^{2} \phi}{\partial \theta_{2}^{2}}+g \frac{\partial \phi}{\partial z} \\
-2\left(\omega_{1} \frac{\partial f}{\partial \theta_{1}}+\omega_{2} \frac{\partial f}{\partial \theta_{2}}\right)+\hat{\nabla} \phi \cdot \hat{\nabla} f .
\end{gathered}
$$

Then the zeroth-order deformation equation can be constructed as

$$
\hat{\nabla}^{2} \breve{\phi}\left(\theta_{1}, \theta_{2}, z ; q\right)=0, \quad-d<z \leq \breve{\eta}\left(\theta_{1}, \theta_{2} ; q\right),
$$

which is subject to the bottom boundary condition

$$
\frac{\partial \breve{\phi}\left(\theta_{1}, \theta_{2}, z ; q\right)}{\partial z}=0, \quad z=-d,
$$


and two nonlinear boundary conditions on $z=\breve{\eta}\left(\theta_{1}, \theta_{2} ; q\right)$ are as follows:

$$
\begin{gathered}
(1-q) \mathrm{L}_{1}\left[\breve{\eta}\left(\theta_{1}, \theta_{2} ; q\right)\right]=q c_{0} \mathrm{~N}_{1}\left[\breve{\eta}\left(\theta_{1}, \theta_{2} ; q\right), \breve{\phi}\left(\theta_{1}, \theta_{2}, z ; q\right)\right], \\
(1-q) \mathrm{L}_{2}\left[\breve{\phi}\left(\theta_{1}, \theta_{2}, z ; q\right)-\phi_{0}\left(\theta_{1}, \theta_{2}, z\right)\right]=q c_{0} \mathrm{~N}_{2}\left[\breve{\phi}\left(\theta_{1}, \theta_{2}, z ; q\right)\right],
\end{gathered}
$$

where $q \in[0,1]$ is an embedding parameter; $c_{0}$ is the so-called nonzero convergence-control parameter; $\phi_{0}\left(\theta_{1}, \theta_{2}, z\right)$ is the initial estimate of the potential function; and $\breve{\phi}\left(\theta_{1}, \theta_{2}, z ; q\right)$ and $\breve{\eta}\left(\theta_{1}, \theta_{2} ; q\right)$ are the mapping functions, respectively.

When $q=0$, the zeroth-order deformation Eqs. (A6)-(A9) have the solution,

$$
\begin{gathered}
\breve{\phi}\left(\theta_{1}, \theta_{2}, z ; 0\right)=\phi_{0}\left(\theta_{1}, \theta_{2}, z\right), \\
\breve{\eta}\left(\theta_{1}, \theta_{2} ; 0\right)=0 .
\end{gathered}
$$

When $q=1$, the zeroth-order deformation Eqs. (A6)-(A9) are equivalent to the original Partial Differential Equations (PDEs) (8)-(11), respectively, provided that

$$
\begin{aligned}
\breve{\phi}\left(\theta_{1}, \theta_{2}, z ; 1\right) & =\phi\left(\theta_{1}, \theta_{2}, z\right), \\
\breve{\eta}\left(\theta_{1}, \theta_{2} ; 1\right) & =\eta\left(\theta_{1}, \theta_{2}\right) .
\end{aligned}
$$

Thus, as the embedding parameter $q$ increases from 0 to $1, \breve{\phi}\left(\theta_{1}, \theta_{2}, z ; q\right)$ and $\breve{\eta}\left(\theta_{1}, \theta_{2} ; q\right)$ deform continuously from initial estimates $\phi_{0}\left(\theta_{1}, \theta_{2}, z\right)$ and 0 to become the exact solutions of the original problem, respectively.

The Maclaurin series of $\breve{\phi}\left(\theta_{1}, \theta_{2}, z ; q\right)$ and $\breve{\eta}\left(\theta_{1}, \theta_{2} ; q\right)$, with respect to the embedding parameter $q$, can be expressed as

$$
\begin{aligned}
\breve{\phi}\left(\theta_{1}, \theta_{2}, z ; q\right) & =\sum_{m=0}^{+\infty} \phi_{m}\left(\theta_{1}, \theta_{2}, z\right) q^{m}, \\
\breve{\eta}\left(\theta_{1}, \theta_{2} ; q\right) & =\sum_{m=0}^{+\infty} \eta_{m}\left(\theta_{1}, \theta_{2}\right) q^{m}
\end{aligned}
$$

where

$$
\begin{aligned}
\phi_{m}\left(\theta_{1}, \theta_{2}, z\right) & =\left.\frac{1}{m !} \frac{\partial^{m} \breve{\phi}\left(\theta_{1}, \theta_{2}, z ; q\right)}{\partial q^{m}}\right|_{q=0}, \\
\eta_{m}\left(\theta_{1}, \theta_{2}\right) & =\left.\frac{1}{m !} \frac{\partial^{m} \breve{\eta}\left(\theta_{1}, \theta_{2} ; q\right)}{\partial q^{m}}\right|_{q=0} .
\end{aligned}
$$

Assuming that $c_{0}$ is properly chosen so that the Maclaurin series (A14) and (A15) converge at $q=1$, then the so-called homotopy-series solutions are obtained as

$$
\begin{gathered}
\phi\left(\theta_{1}, \theta_{2}, z\right)=\phi_{0}\left(\theta_{1}, \theta_{2}, z\right)+\sum_{m=1}^{+\infty} \phi_{m}\left(\theta_{1}, \theta_{2}, z\right), \\
\eta\left(\theta_{1}, \theta_{2}\right)=\sum_{m=1}^{+\infty} \eta_{m}\left(\theta_{1}, \theta_{2}\right) .
\end{gathered}
$$

\section{High-order deformation equation}

Substituting the series in Eqs. (A14) and (A15) into the zeroth-order deformation equations and equating the like-power of $q$, the so-called $m$ th-order deformation equations are

$$
\begin{gathered}
\hat{\nabla}^{2} \phi_{m}\left(\theta_{1}, \theta_{2}, z\right)=0, \\
\frac{\partial \phi_{m}\left(\theta_{1}, \theta_{2}, z ; q\right)}{\partial z}=0, \quad z=-d, \\
\overline{\mathrm{L}}_{2}\left[\phi_{m}\left(\theta_{1}, \theta_{2}, z\right)\right]=\mathrm{R}_{m}^{\phi}\left(\theta_{1}, \theta_{2} ; c_{0}\right),
\end{gathered}
$$




$$
\eta_{m}\left(\theta_{1}, \theta_{2}\right)=\mathrm{R}_{m}^{\eta}\left(\theta_{1}, \theta_{2} ; c_{0}\right)
$$

where

$$
\begin{gathered}
\mathrm{R}_{m}^{\phi}\left(\theta_{1}, \theta_{2} ; c_{0}\right)=c_{0} \Delta_{m-1}^{\phi}+\chi_{m} S_{m-1}-\bar{S}_{m}, \\
\mathrm{R}_{m}^{\eta}\left(\theta_{1}, \theta_{2} ; c_{0}\right)=c_{0} \Delta_{m-1}^{\eta}+\chi_{m} \eta_{m-1}, \\
\Delta_{m}^{\phi}=\omega_{1}^{2} \bar{\phi}_{m}^{2,0}+2 \omega_{1} \omega_{2} \bar{\phi}_{m}^{1,1}+\omega_{2}^{2} \bar{\phi}_{m}^{0,2}+g \bar{\phi}_{z, m}^{0,0}-2\left(\omega_{1} \Gamma_{m, 1}+\omega_{2} \Gamma_{m, 2}\right)+\Lambda_{m}, \\
\Delta_{m}^{\eta}=\eta_{m}-\frac{1}{g}\left(\omega_{1} \bar{\phi}_{m}^{1,0}+\omega_{2} \bar{\phi}_{m}^{0,1}-\Gamma_{m, 0}\right),
\end{gathered}
$$

$\overline{\mathrm{L}}_{2}[\phi]=\left.\mathrm{L}_{2}[\phi]\right|_{z=0}$ and $m \geq 1$. The definitions of $S_{m}, \bar{S}_{m}, \chi_{m}, \Lambda_{m}, \bar{\phi}_{z, m}^{0,0}, \Gamma_{m, i}, \bar{\phi}_{m}^{i, j} \quad(i, j=0,1,2)$ and their detailed derivations can be found in the work of Liao. ${ }^{15}$

\section{The initial estimate}

Liao $^{12}$ has demonstrated that there is great freedom to choose the initial estimate in HAM. The auxiliary linear operator in Eq. (A2) has the property,

$$
\overline{\mathrm{L}}_{2}\left[\Psi_{m, n}\right]=\lambda_{m, n} \cdot \sin \left(m \theta_{1}+n \theta_{2}\right),
$$

where $\Psi_{m, n}$ is defined by Eq. (16) and

$$
\lambda_{m, n}=g\left|m k_{1}+n k_{2}\right| \tanh \left(\left|m k_{1}+n k_{2}\right| d\right)-\left(m \bar{\omega}_{1}+n \bar{\omega}_{2}\right)^{2} .
$$

Therefore, the inverse operator $\overline{\mathrm{L}}_{2}^{-1}$ is defined as

$$
\overline{\mathrm{L}}_{2}^{-1}\left[\sin \left(m \theta_{1}+n \theta_{2}\right)\right]=\frac{\Psi_{m, n}}{\lambda_{m, n}}, \quad \lambda_{m, n} \neq 0 .
$$

Note that the inverse operator $\overline{\mathrm{L}}_{2}^{-1}$ has definition only for non-zero values of $\lambda_{m, n}$. When $\lambda_{m, n}=0$,

$$
g\left|m k_{1}+n k_{2}\right| \tanh \left(\left|m k_{1}+n k_{2}\right| d\right)=\left(m \bar{\omega}_{1}+n \bar{\omega}_{2}\right)^{2} .
$$

In this paper, there are only $\lambda_{1,0}=0$ and $\lambda_{0,1}=0$. Thus, an initial estimate for $\phi_{0}\left(\theta_{1}, \theta_{2}, z\right)$ can be chosen as

$$
\phi_{0}\left(\theta_{1}, \theta_{2}, z\right)=b_{1,0} \cdot \Psi_{1,0}\left(\theta_{1}, \theta_{2}, z\right)+b_{0,1} \cdot \Psi_{0,1}\left(\theta_{1}, \theta_{2}, z\right),
$$

where $b_{1,0}$ and $b_{0,1}$ are unknown constants to be determined later.

\section{Solution procedure}

Considering the rule for solution expressions (14) and (15) and the property of the auxiliary linear operator $\mathrm{L}_{2}$ in Eq. (A28), the right-hand side of Eq. (A22) can be expressed as

$$
\mathrm{R}_{m}^{\phi}=\tilde{b}_{m, 1,0} \sin \theta_{1}+\tilde{b}_{m, 0,1} \sin \theta_{2}+\sum_{i=0}^{I_{m}} \sum_{\substack{j=-J_{m} \\ i+j \neq 1}}^{J_{m}} \tilde{b}_{m, i, j} \sin \left(i \theta_{1}+j \theta_{2}\right),
$$

where $\tilde{b}_{m, i, j}$ are coefficients and $\left(I_{m}, J_{m}\right)$ is related to the right-hand side of Eq. (A22). According to the property of the auxiliary linear operator $\mathrm{L}_{2}$,

$$
\left\{\begin{array}{l}
\tilde{b}_{m, 1,0}=0 \\
\tilde{b}_{m, 0,1}=0
\end{array}\right.
$$

have to be enforced to avoid the so-called secular terms. Therefore, using Eq. (A31), it is convenient to obtain the solution of Eq. (A22)

$\phi_{m}\left(\theta_{1}, \theta_{2}, z\right)=\sum_{i=0}^{I_{m}} \sum_{\substack{j=-J_{m} \\ i+j \neq 1}}^{J_{m}} \bar{b}_{m, i, j} \Psi_{m, n}\left(\theta_{1}, \theta_{2}, z\right)+\bar{b}_{m, 1,0} \Psi_{1,0}\left(\theta_{1}, \theta_{2}, z\right)+\bar{b}_{m, 0,1} \Psi_{0,1}\left(\theta_{1}, \theta_{2}, z\right)$, 
where $\bar{b}_{m, 1,0}$ and $\bar{b}_{m, 0,1}$ are unknown coefficients to be determined in the $(m+1)$ th-order deformation equation.

When $m=1$ using Eq. (A34), the unknown coefficients $b_{1,0}$ and $b_{0,1}$ in Eq. (A32) can be obtained for the initial estimate $\phi_{0}\left(\theta_{1}, \theta_{2}, z\right)$. Substituting $\phi_{0}\left(\theta_{1}, \theta_{2}, z\right)$ into Eq. (A23), $\eta_{1}\left(\theta_{1}, \theta_{2}\right)$ can be directly obtained.

When $m \geq 2$, the unknown coefficients $\bar{b}_{m-1,1,0}$ and $\bar{b}_{m-1,0,1}$ in Eq. (A35) can also be obtained by using Eq. (A34). As long as $\bar{b}_{m-1,1,0}$ and $\bar{b}_{m-1,0,1}$ are known, $\eta_{m}\left(\theta_{1}, \theta_{2}\right)$ and $\phi_{m}\left(\theta_{1}, \theta_{2}, z\right)$ can be obtained in a similar way. All of this can be done successively and efficiently by means of the symbolic computation software-Mathematica 7 . At the $m$ th - order approximations, there are

$$
\left\{\begin{array}{l}
\phi\left(\theta_{1}, \theta_{2}, z\right) \approx \phi_{0}\left(\theta_{1}, \theta_{2}, z\right)+\sum_{m=1}^{M} \phi_{m}\left(\theta_{1}, \theta_{2}, z\right), \\
\eta\left(\theta_{1}, \theta_{2}\right) \approx \sum_{m=1}^{M} \eta_{m}\left(\theta_{1}, \theta_{2}\right) .
\end{array}\right.
$$

\section{Optimal convergence-control parameters}

For the $m$ th-order approximations $\phi\left(\theta_{1}, \theta_{2}, z\right)$ and $\eta\left(\theta_{1}, \theta_{2}\right)$, there is still one unknown parameter $c_{0}$, which is used to guarantee the convergence of the approximation series. In order to choose an optimal $c_{0}$, two averaged residual square errors of the boundary conditions are defined as

$$
\begin{gathered}
\mathrm{E}_{m}^{\phi}=\frac{1}{\left(1+I_{k}\right)} \frac{1}{\left(1+J_{k}\right)} \sum_{i=0}^{I_{k}} \sum_{j=0}^{J_{k}}\left(\left.\mathrm{~N}_{1}\left[\phi\left(\theta_{1}, \theta_{2}, z\right)\right]\right|_{\theta_{1}=i \Delta \theta_{1}, \theta_{2}=j \Delta \theta_{2}}\right)^{2}, \\
\mathrm{E}_{m}^{\eta}=\frac{1}{\left(1+I_{k}\right)} \frac{1}{\left(1+J_{k}\right)} \sum_{i=0}^{I_{k}} \sum_{j=0}^{J_{k}}\left(\left.\mathrm{~N}_{2}\left[\phi\left(\theta_{1}, \theta_{2}, z\right), \eta\left(\theta_{1}, \theta_{2}\right)\right]\right|_{\theta_{1}=i \Delta \theta_{1}, \theta_{2}=j \Delta \theta_{2}}\right)^{2},
\end{gathered}
$$

where $I_{k}$ and $J_{k}$ are the numbers of discrete points, $\Delta \theta_{1}=\pi / I_{k}$ and $\Delta \theta_{2}=\pi / J_{k}$. In the present work, $I_{k}=J_{k}=20$ is chosen based on the sensitivity test without loss of generality. Defining the total averaged residual square error as $\mathrm{E}_{m}^{T}=\mathrm{E}_{m}^{\phi}+\mathrm{E}_{m}^{\eta}$, then by solving $\mathrm{dE}_{m}^{T} / \mathrm{d} c_{0}=0$, the optimal value of $c_{0}$ can be obtained, which corresponds to the minimum value of $\mathrm{E}_{m}^{T}$.

${ }^{1}$ O. M. Phillips, “On the dynamics of unsteady gravity waves of finite amplitude Part 1 . The elementary interactions,” J. Fluid Mech. 9, 193 (1960).

${ }^{2}$ M. S. Longuet-Higgins, "Resonant interactions between two trains of gravity waves," J. Fluid Mech. 12, 321 (1962)

${ }^{3}$ W. J. Pierson, "Oscillatory third-order perturbation solutions for sums of interacting long-crested Stokes waves on deep water," J. Ship Res. 37, 354 (1993).

4 J. F. Dalzell, "A note on finite depth second-order wave-wave interactions," Appl. Ocean Res. 21, 105 (1999).

${ }^{5}$ T. Ohyama, D.-S. Jeng, and J. R. C. Hsu, "Fourth-order theory for multiple-wave interaction," Coastal Eng. 25, 43 (1995).

${ }^{6}$ L. Chen and J. Zhang, "On interaction between intermediate-depth long waves and deep-water short waves," Ocean Eng. 25, 395 (1998).

${ }^{7}$ J. Zhang and L. Chen, "General third-order solutions for irregular waves in deep water," J. Eng. Mech. 125, 768 (1999).

${ }^{8}$ P. A. Madsen and D. R. Fuhrman, "Third-order theory for bichromatic bi-directional water waves," J. Fluid Mech. 557, 369 (2006).

${ }^{9}$ T. S. Jang and S. H. Kwon, "Application of nonlinear iteration scheme to the nonlinear water wave problem: Stokian wave," Ocean Eng. 32, 1864 (2005)

${ }^{10}$ T. S. Jang, S. H. Kwon, and H. S. Choi, "Nonlinear wave profiles of wave-wave interaction in a finite water depth by fixed point approach," Ocean Eng. 34, 451 (2007).

${ }^{11}$ Z. Lin, L. Tao, Y. C. Pu, and A. J. Murphy, "Fully nonlinear solution of bi-chromatic deep-water waves," Ocean Eng. 91, 290 (2014).

${ }^{12}$ S. Liao, Beyond Perturbation: Introduction to the Homotopy Analysis Method (Chapman and Hall, London/CRC, Boca Raton, FL, 2003).

${ }^{13}$ S. Liao and K. Cheung, "Homotopy analysis of nonlinear progressive waves in deep water," J. Eng. Math. 45, 105 (2003)

${ }^{14}$ L. Tao, H. Song, and S. Chakrabarti, "Nonlinear progressive waves in water of finite depth-An analytic approximation," Coastal Eng. 54, 825 (2007).

${ }^{15}$ S. Liao, "On the homotopy multiple-variable method and its applications in the interactions of nonlinear gravity waves," Commun. Nonlinear Sci. Numer. Simul. 16, 1274 (2011).

${ }^{16}$ D. Xu, Z. Lin, S. Liao, and M. Stiassnie, “On the steady-state fully resonant progressive waves in water of finite depth,” J. Fluid Mech. 710, 379 (2012). 
${ }^{17}$ Z. Liu and S. Liao, "Steady-state resonance of multiple wave interactions in deep water," J. Fluid Mech. 742, 664 (2014).

${ }^{18}$ A. D. D. Craik, Wave Interactions and Fluid Flows (Cambridge University Press, Cambridge, 1985).

${ }^{19}$ R. E. Baddour and S. W. Song, "Interaction of higher-order water waves with uniform currents," Ocean Eng. 17, 551 (1990)

${ }^{20}$ R. E. Baddour and S. Song, "On the interaction between waves and currents," Ocean Eng. 17, 1 (1990),

${ }^{21}$ M. H. Zaman and E. Baddour, "Interaction of waves with non-colinear currents," Ocean Eng. 38, 541 (2011).

${ }^{22}$ Y. Ma, G. Dong, and X. Ma, "Separation of low frequency waves by an analytical method," in Proceedings of the 32th international conference on coastal engineering, (ICCE) (ASCE, Shanghai, 2010).

${ }^{23}$ G. B. Whitham, "Mass, momentum and energy flux in water waves," J. Fluid Mech. 12, 135 (1962). 